\title{
Sodium arsenite accelerates TRAIL-mediated apoptosis in melanoma cells through upregulation of TRAIL-R1/R2 surface levels and downregulation of $c F L I P$ expression
}

\author{
Vladimir N. Ivanov ${ }^{a, *}$, Tom K. Hei ${ }^{a, b}$ \\ ${ }^{a}$ Center for Radiological Research, Columbia University College of Physicians and Surgeons, Columbia University, New York, NY 10032, USA \\ ${ }^{b}$ Department of Environmental Health Sciences, Mailman School of Public Health, Columbia University, New York, NY 10032, USA
}

\section{A R T I C L E I N F O R M A T I O N}

Article Chronology:

Received 17 July 2006

Revised version received

23 August 2006

Accepted 13 September 2006

Available online 28 September 2006

Keywords:

Apoptosis

cJun

TRAIL

TRAIL-R

CFLIP

Melanoma

\begin{abstract}
A B S T R A C T
AP-1/cJun, NF- $\kappa$ B and STAT3 transcription factors control expression of numerous genes, which regulate critical cell functions including proliferation, survival and apoptosis. Sodium arsenite is known to suppress both the IKK-NF-KB and JAK2-STAT3 signaling pathways and to activate the MAPK/JNK-cJun pathways, thereby committing some cancers to undergo apoptosis. Indeed, sodium arsenite is an effective drug for the treatment of acute promyelocytic leukemia with little nonspecific toxicity. Malignant melanoma is highly refractory to conventional radio- and chemotherapy. In the present study, we observed strong effects of sodium arsenite treatment on upregulation of TRAIL-mediated apoptosis in human and mouse melanomas. Arsenite treatment upregulated surface levels of death receptors, TRAIL-R1 and TRAIL-R2, through increased translocation of these proteins from cytoplasm to the cell surface. Furthermore, activation of cJun and suppression of NF- $\kappa$ B by sodium arsenite resulted in upregulation of the endogenous TRAIL and downregulation of the cFLIP gene expression (which encodes one of the main anti-apoptotic proteins in melanomas) followed by cFLIP protein degradation and, finally, by acceleration of TRAILinduced apoptosis. Direct suppression of cFLIP expression by CFLIP RNAi also accelerated TRAIL-induced apoptosis in these melanomas, while COX-2 suppression substantially increased levels of both TRAIL-induced and arsenite-induced apoptosis. In contrast, overexpression of permanently active AKTmyr inhibited TRAIL-mediated apoptosis via downregulation of TRAIL-R1 levels. Finally, AKT overactivation increased melanoma survival in cell culture and dramatically accelerated growth of melanoma transplant in vivo, highlighting a role of AKT suppression for effective anticancer treatment.
\end{abstract}

(c) 2006 Elsevier Inc. All rights reserved.

\footnotetext{
* Corresponding author. Center for Radiological Research, Columbia University, VC11-204, 630 West 168th Street, New York, NY 10032, USA. Fax: +12123053229 .

E-mail address: vni3@columbia.edu (V.N. Ivanov).
} 


\author{
Abbreviations: \\ Ac-IETD-CHO, N-acetyl-Ile-Glu-Thr- \\ Asp-CHO (aldehyde) \\ Ac-LEHD-CHO, N-acetyl-Leu-Glu- \\ His-Asp-CHO (aldehyde) \\ AKTmyr, myristoylated AKT \\ AP-1, activator protein 1 \\ APAF-1, apoptotic protease- \\ associated factor-1 \\ ATF2, activating transcription \\ factor 2 \\ CHX, cycloheximide \\ COX-2, cyclooxygenase-2 \\ EMSA, electrophoretic mobility \\ shift assay \\ ERK, extracellular signal-regulated \\ kinase \\ FACS, fluorescence-activated \\ cell sorter \\ FasL, Fas Ligand \\ FLIP, FLICE inhibitory protein \\ hFLIP, human FLIP \\ cFLIP $_{\mathrm{L}}$, cellular FLIP long \\ cFLIP $_{\mathrm{S}}$, cellular FLIP short \\ I $\mathrm{B}$, inhibitor of NF- $\mathrm{kB}$ \\ IKK, inhibitor nuclear factor kappa \\ $B$ kinase \\ JNK, Jun N-terminal kinase \\ $\mathrm{mAb}$, monoclonal $\mathrm{Ab}$ \\ Luc, luciferase \\ MAPK, mitogen-activated \\ protein kinase \\ MEK, MAPK kinase \\ MEKK1, MAPK/ERK kinase, \\ kinase-1 \\ MFI, medium fluorescence intensity \\ NF- $\mathrm{B}$, nuclear factor kappa B \\ PARP, poly(ADP-ribose) polymerase \\ PI, propidium iodide \\ PI3K, phosphatidylinositol 3-kinase \\ ROS, reactive oxygen species \\ RNAi, RNA interference \\ STAT, signal transducer and \\ activator of transcription \\ STAT3-C, constitutively activated \\ STAT3 \\ STAT3-F, DNA-binding mutant \\ of STAT3 \\ TNF $\alpha$, tumor necrosis factor alpha \\ TNFR, tumor necrosis factor receptor \\ TRAIL, TNF-related apoptosis- \\ inducing ligand \\ hTRAIL, human TRAIL \\ TRAIL-R, TRAIL receptor \\ XIAP, X-linked inhibitor of apoptosis \\ zVAD-fmk, benzyloxycarbonyl-Val- \\ Ala-Asp-fluoromethyl ketone
}




\section{Introduction}

Malignant melanoma remains one of the most difficult types of cancer for successful treatment, and the incidence of melanoma has been progressively increasing over the last 40 years throughout the world [1]. The impairment of apoptosis is one of the key characteristics of cancer development, which renders tumors resistant to cytotoxic therapy [2]. Consequently, anticancer drug discovery strategies have focused on core components of the apoptosis machinery in the cell, as main targets, in order to restore sensitivity of cancer cells to apoptosis [3]. In this respect, the use of arsenic trioxide (or sodium arsenite) as a regulator of gene expression and a stimulator of oxidative stress, which was followed by induction of programmed cell death, has been shown to be effective in the treatment of acute promyelocytic leukemia (APL) [4] and of prostate cancer cell lines [5]. Arsenic trioxide was approved by the U.S. Food and Drug Administration for the treatment of relapsed APL that is refractory to therapy with all-trans retinoic acid [Food and Drug Administration (2000), FDA approves arsenic trioxide for leukemia treatment (http://www.fda.gov/ bbs/topics/ANSWERS/ANS01040.html)]. However, a phase II trial of arsenic trioxide in patients with metastatic melanoma was unsuccessful indicating that subsequent clinical trials should evaluate arsenic in combinations with other anticancer drugs that may increase its clinical efficacy [6]. We and others have recently demonstrated a profound increase in apoptosis of human melanomas when treated with a combination of sodium arsenite and an inhibitor of an additional survival pathway (EGFR, MEK-ERK, PI3K-AKT and COX-2) [7-9] that was accompanied by the profound increase in cancer cell apoptosis.

Tumor necrosis factor alpha-related apoptosis-inducing ligand (TRAIL; APO2L; TNFSF10) is a member of the TNF superfamily of death receptor ligands and has exhibited great therapeutic potential against different types of tumors [10]. However, TRAIL is not a universal anticancer agent because many types of cancer cells still possess resistance to TRAIL. For human melanoma, cell lines both sensitive and resistant to TRAIL have been described [11-14]. TRAIL induces the death signaling cascades by binding one of two cell surface receptors, TRAIL-R1/DR4/TNFRSF10A or TRAIL-R2/DR5/ TNFRSF10B. Once ligand bound, these receptors assemble a death-inducing signaling complex (DISC) that contains an adaptor protein FADD, which recruits the apoptosis-initiating caspase-8/caspase-10. After processing and activation, these caspases directly, or through the mitochondrial loop, target executive caspases [2,15]. In addition, numerous anti-apoptotic proteins (such as cFLIP, cIAP1/2, XIAP, survivin, Bcl2 and BclxL) negatively regulate the development of apoptotic signaling $[2,16]$. Differential splicing of cFLIP/CFLAR gene may produce several isoforms, however, cFLIP $_{\mathrm{L}}$ (55-kDa-protein) and $\operatorname{cFLIP}_{\mathrm{S}}(25 \mathrm{kDa})$ are the main products. There is a close structural similarity between CFLIP $_{\mathrm{L}}$ and caspase-8; furthermore, CFLIP $_{\mathrm{L}}$ binds to caspase- 8 at the DISC and can effectively block its activation [17]. Recent investigations have further confirmed an anti-apoptotic role of $\operatorname{CFLIP}_{\mathrm{L}}[18,19]$, although some other observations indicated that, in certain conditions, the long form of cFLIP may also support caspase-8 activation
[20]. The role of the short form, cFLIP , as an inhibitor of death receptor-mediated apoptosis is well established [21].

An additional level for attenuation of TRAIL-mediated signaling is based on decoy receptors TRAIL-R3 and TRAILR4, which are capable of binding TRAIL but do not transmit death signals, which decreases the efficiency of apoptosis induction [10]. Hence, an effective initiation and progression of the TRAIL/TRAIL-R-mediated signaling in cancer cells requires: (i) an exogenous ligand (as a recombinant soluble protein or as a membrane protein on the surface of killer cells) or an induction of the endogenous surface expression of TRAIL in the population of target cells; (ii) appropriate levels of TRAIL-R1/R2 on the cell surface, efficient death signaling induced by TRAIL-mediated receptor oligomerization and the DISC assembling; and (iii) effective repression of anti-apoptotic protein activities in target cells. This multifaceted approach for cancer cell treatment may be achieved in some cases using combined treatment of soluble recombinant TRAIL together with specific inhibitors of cell survival pathways or with specific suppressors of anti-apoptotic proteins.

Many human melanomas express different types of death receptors, such as Fas/APO1, TNF-R1 and TRAIL-R1/R2 (DR4/ DR5) on the cell surface [11-13,22]. Recombinant soluble Fas ligand (FasL/APO1-L) or agonistic anti-Fas monoclonal antibody $(\mathrm{mAb})$ can induce apoptosis in Fas-positive melanomas with varying efficiency, depending on levels of surface Fas expression and the effectiveness of death signaling. However, the strong toxicity of soluble FasL diminishes efficacy of this treatment for in vivo applications. In contrast, soluble recombinant TRAIL/APO2-L is relatively nontoxic after systemic treatment as demonstrated in numerous experiments with different types of tumors in vivo [10], although distinct recombinant versions of TRAIL demonstrated differential hepatotoxicity.

In the present study, we have used sodium arsenite as a modulator of cell signaling pathways to overcome the resistance of melanoma cell lines to TRAIL. We have demonstrated strong positive effects of sodium arsenite on TRAILmediated apoptosis of some melanoma lines and have determined conditions that allow the efficient upregulation of TRAIL-R levels, induction of the endogenous TRAIL expression, suppression of anti-apoptotic proteins and development of TRAIL/TRAIL-R-mediated apoptosis.

\section{Methods}

\section{Materials}

Sodium arsenite and cycloheximide were obtained from Sigma (St. Louis, MO). Human soluble Fas ligand (recombinant) and soluble Killer-TRAIL (recombinant) were purchased from Alexis (San Diego, CA). IKK inhibitor BAY11-7082 and JNK inhibitor SP600125 were obtained from Biomol (Plymouth Meeting, PA); MEK inhibitor U0126 and PI3K inhibitor LY294002 were purchased from Calbiochem (La Jolla, CA). Caspase inhibitors zVAD-fmk, Ac-IETD-CHO (an inhibitor of caspase-8 and caspase-6) and Ac-LEHD-CHO (an inhibitor of caspase-9) were purchased from Calbiochem (La Jolla, CA). 


\section{Cell lines}

Human melanoma cell lines LU1205 (also known as 1205lu), WM793, WM9, WM35, SBCl2 [23] and OM431 as well as mouse melanoma K1735-SW1 were maintained in a DMEM medium supplemented with $10 \%$ fetal bovine serum (FBS), L-glutamine and antibiotics. FEMX, LOX and HHMSX human melanoma lines were maintained in an RPMI1640 medium supplemented with $10 \%$ FBS and antibiotics.

\section{FACS analysis of TRAIL and TRAIL-R1/R2 levels}

Surface levels of TRAIL on human and mouse melanomas were determined by staining with the PE-conjugated antihuman-TRAIL or anti-mouse-TRAIL mAbs and subsequent flow cytometry. PE-conjugated mAbs were obtained from eBioscience (San Diego, CA). Surface levels of TRAIL-R1/DR4 and TRAIL-R2/DR5 were determined by staining with the corresponding primary mAbs followed by staining with antimouse PE-IgG (BD Biosciences Pharmingen, San Jose, CA). PEconjugated nonspecific mouse IgG1 was used as an immunoglobulin isotype control. A FACS Calibur flow cytometer (Becton Dickinson, Mountain View, CA) combined with the CellQuest program was used to perform flow cytometric analysis. All experiments were independently repeated 4-5 times.

\section{Transfection and luciferase assay}

The NF- $\kappa$ B luciferase reporter containing two $\kappa$ B-binding sites, Jun2-Luc reporter and empty vector tk-Luc [24], GAS-Luc reporter containing three repeats of GAS sites from the Ly6A/E promoter [25] were used to determine NF-кB, AP-1 and STAT transactivation, respectively. Additional reporter constructs used included $1.5 \mathrm{~kb}$ TRAILpr-Luc [26], $1.8 \mathrm{~kb}$ TRAILR1pr-Luc [27] and $1 \mathrm{~kb}$ cFLIPpr-Luc [28,29]. Transient transfection of different reporter constructs $(1 \mu \mathrm{g})$ together with pCMV$\beta$ gal $(0.25 \mu \mathrm{g})$ into $5 \times 10^{5}$ melanoma cells was performed using Lipofectamine (Life Technologies, Invitrogen). Proteins were prepared for $\beta$-Gal and luciferase analysis $16 \mathrm{~h}$ after transfection. Luciferase activity was determined using the Luciferase assay system (Promega, Madison, WI) and was normalized based on $\beta$-galactosidase levels.

In some experiments, melanoma cells were transfected with reporter constructs together with certain expression vectors (ratio 1:3), including $\mathrm{pCMV-I \kappa B} \alpha \Delta \mathrm{N}$ [30], pcDNA3IKK $\beta$ S178E/S181E [31], $\triangle$ MEKK1 expression vector [32], pcDNA3-FLAG-MKK7ß1 [33], dominant-negative JNK1-APF [34], dominant-negative form of cJun/TAM67 [35], dominantnegative STAT3- $\beta$ [36], dominant-negative STAT3-F and permanently active STAT3-C [37] in the presence of pCMV$\beta \mathrm{Gal}$. Sixteen to $24 \mathrm{~h}$ after transfection, luciferase activity was determined.

\section{Apoptosis studies}

Cells were exposed to soluble TRAIL (50 ng/ml) alone or in combination with cycloheximide $(2 \mu \mathrm{g} / \mathrm{mL})$ or sodium arsenite $(4 \mu \mathrm{M})$. Apoptosis was then assessed by quantifying the percentage of hypodiploid nuclei undergoing DNA fragmenta- tion. Flow cytometric analysis was performed on a FACS Calibur flow cytometer.

\section{cFLIP and COX-2 suppression by RNAi}

The pSUPER retro RNA interference (RNAi) system (Oligoengine, Seattle, WA), which has been utilized for the production of small RNAi transcripts used to suppress cFLIP and COX-2 expression. Three variants of RNAi of 19 nucleotides designed to target human COX-2 mRNA and two variants of RNAi to target human cFLIP were expressed using vector pSUPER.retro. puro (pSR-puro). RNAi cFLIP-92 (UGUGGUUCCACCUAAUGUC) and RNAi COX-2-379 (CCUUCUCUAACCUCUCCUA) were the most efficient in the corresponding mRNA targeting.

\section{Western blot analysis}

Total cell lysates (50-100 $\mu$ g protein) were resolved on $10 \%$ SDS-PAGE, and processed according to standard protocols. The antibodies (Abs) used for Western blotting included monoclonal anti- $\beta$-Actin (Sigma); monoclonal anti-Caspase-8 and anti-Caspase-3; (Cell Signaling, Beverly, MA); monoclonal anti-Caspase-3 (procaspase and active forms) (Axxora, San Diego, CA); monoclonal anti-FLIP (Dave-2); monoclonal anti-FLIP (NF6) (Axxora, San Diego, CA); monoclonal antiXIAP (BD Biosciences, San Jose, CA); monoclonal anti-COX-2 (Cayman Chemical Company, Ann Arbor, Michigan); polyclonal Abs to TRAIL (human), TRAIL-R1 and TRAIL-R2/DR5 (Axxora, San Diego, CA); polyclonal Abs against: phosphocJun (Ser73) and total cJun; phospho-SAPK/JNK (Thr183/ Tyr185) and JNK; phospho-p44/p42 MAP kinase (Thr202/ Tyr204) and p44/p42 MAP kinase; phospho-p38 MAP kinase (Thr180/Tyr182) and p38 MAP kinase; phospho-STAT3 (Tyr705) and STAT3; phospho-AKT (Ser473) and AKT; phos-

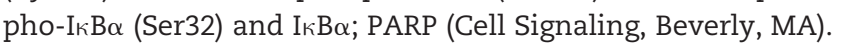
Optimal dilutions of primary Abs were 1:1000 to 1:10000. The secondary Abs (anti-rabbit, anti-mouse or anti-rat) were conjugated to horseradish peroxidase (dilution 1:5000 to 1:10000); signals were detected using the ECL system (Amersham, Piscataway, NJ).

\section{EMSA}

Electrophoretic mobility shift assay (EMSA) was performed for the detection of NF- $\kappa$ B DNA-binding activity as previously described, using the labeled double-strand oligonucleotide AGCTTGGGGACTTTCCAGCCG (binding site is underlined). Ubiquitous NF-Y DNA-binding activity was used as an internal control [7].

\section{Results}

Induction of cell death in melanomas by treatment with recombinant TRAIL and sodium arsenite

Since treatment of cancer cells with combined modality is, as a rule, more effective than monotherapy, our initial aim was to use different combinations of soluble TRAIL with inhibitors of cell survival pathways to upregulate TRAIL-mediated 
apoptosis of sensitive melanoma cells. Based on proapoptotic activities of sodium arsenite [7], we decided to elucidate effects of a combination of TRAIL and sodium arsenite for the acceleration of apoptosis. First we confirmed that normal human melanocytes and normal lung fibroblasts are resistant to cytotoxic effects of sodium arsenite at the dose 1-10 $\mu \mathrm{M}$ (data not shown). These are clinically relevant doses as the arsenic level of $7 \mu \mathrm{M}$ has been reported in the serum of
A
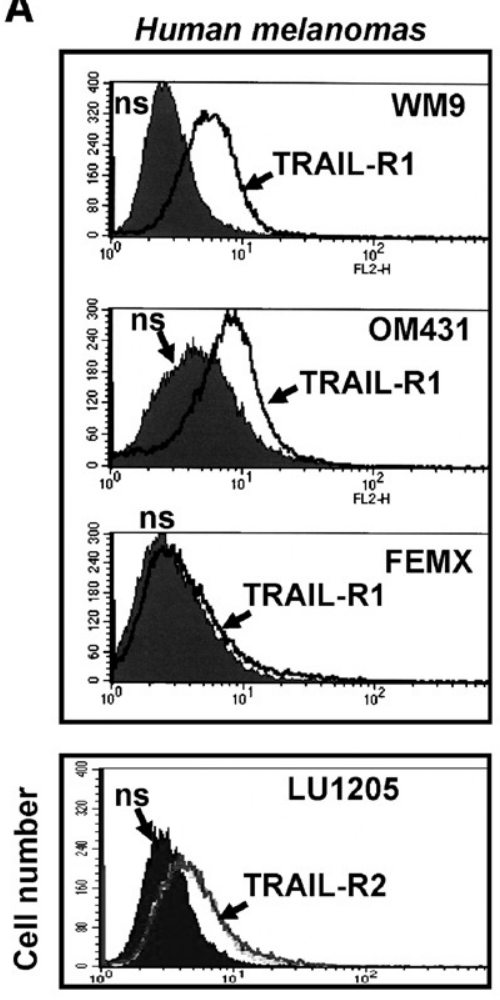

Surface levels

C

WM9 cells

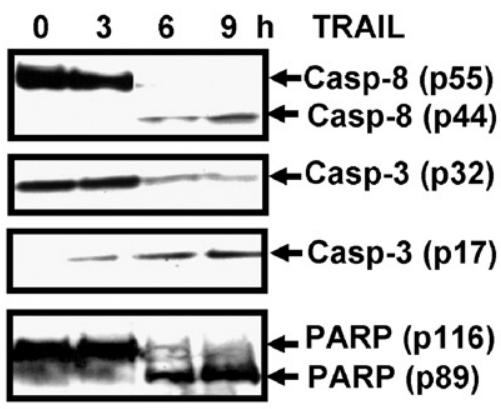

B

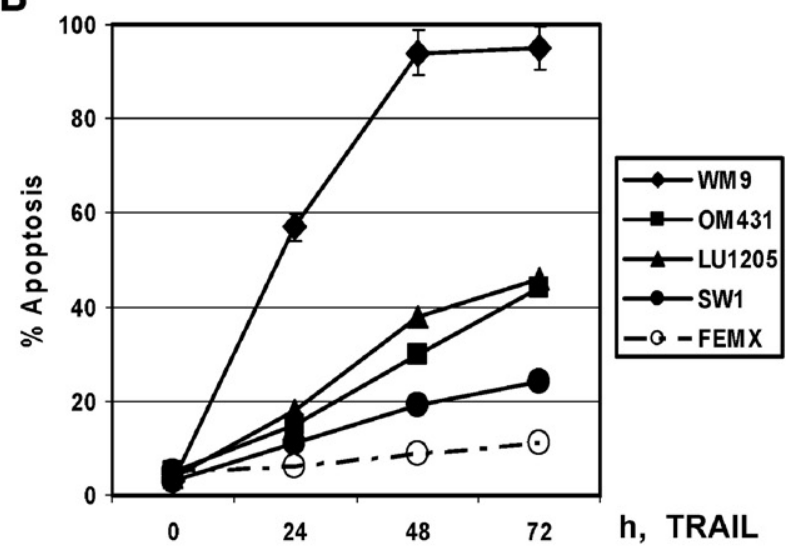

D

WM9 0 OM431 운 LU1205

$24 \mathrm{~h}$

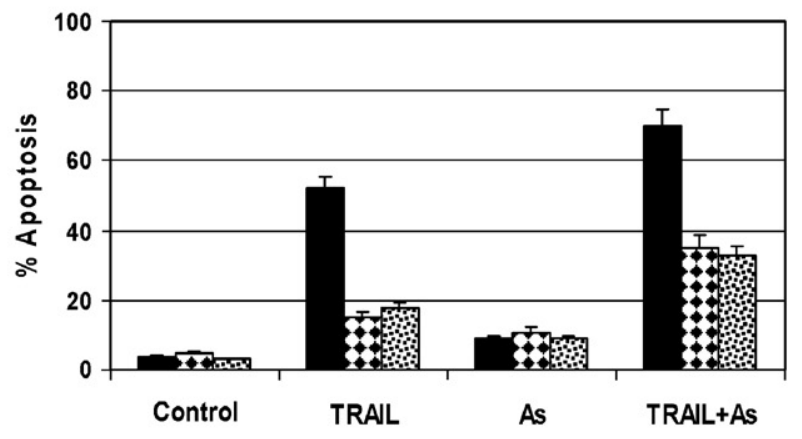

$\mathbf{E}$

$48 \mathrm{~h}$

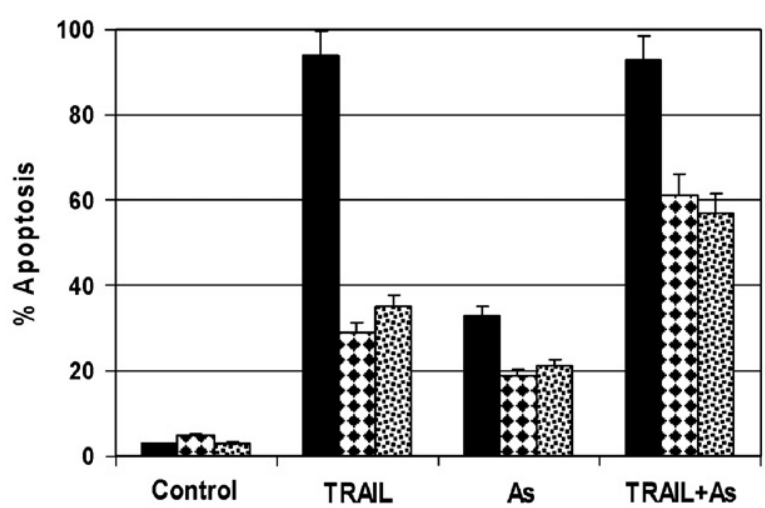

Fig. 1 - TRAIL induces apoptosis in human melanomas. (A) Surface expression of TRAIL-R1 and TRAIL-R2 was determined by staining with PE-labeled anti-TRAIL-R1 (DR4) or anti-TRAIL-R2 (DR5) mAbs, respectively, and FACS analysis. (B) Kinetics of the TRAIL-induced apoptosis in indicated melanoma cell lines. Cells were treated with soluble recombinant TRAIL (50 ng/ml) for indicated time. For apoptosis analysis, cells were stained by PI 24-72 $\mathrm{h}$ after treatment. Levels of apoptosis were determined as percentage of cells with hypodiploid content of DNA in the pre-G0/G1 region using flow cytometry. Error bars represent mean \pm SD from three independent experiments. (C) Western blot analysis of caspase-8 and caspase-3 processing (p17 is active form of caspase-3) and PARP cleavage following treatment of WM9 melanoma cells with TRAIL (50 ng/ml). (D, E) Effects of sodium arsenite (As, $4 \mu \mathrm{M}$ ) on TRAIL-induced apoptosis in WM9, OM431 and LU1205 cells $24 \mathrm{~h}$ and $48 \mathrm{~h}$ after treatment. Error bars represent mean \pm SD from three independent experiments. 
patients $10 \mathrm{~h}$ after treatment with a 10-mg dose, effective for acute promyelocytic leukemia (APL) $[4,38]$. In general, we observed four types of responses in melanomas to sodium arsenite treatment. Early radial growth phase melanomas SBCl2 and WM35, similarly to melanocytes, were relatively resistant to arsenite treatment. Human metastatic melanomas LU1205, WM9 and OM431 developed a low-level apoptosis $24 \mathrm{~h}$ after arsenite treatment, which showed modest increase after $48 \mathrm{~h}$ treatment. A vertical growth phase melanoma WM793 and metastatic melanoma FEMX were highly sensitive to sodium arsenite due to an induction of the TNF $\alpha$-mediated apoptosis that was also accompanied by the secondary necrosis [7]. In contrast, LOX and HHMSX metastatic melanomas demonstrated G2/M arrest $24 \mathrm{~h}$ after arsenite treatment, which was accompanied by low-level apoptosis $48 \mathrm{~h}$ after treatment (data not shown). Hence, arsenite alone at clinically relevant doses $(1-10 \mu \mathrm{M})$ was not a universal and efficient inducer of cell death in melanomas.
A

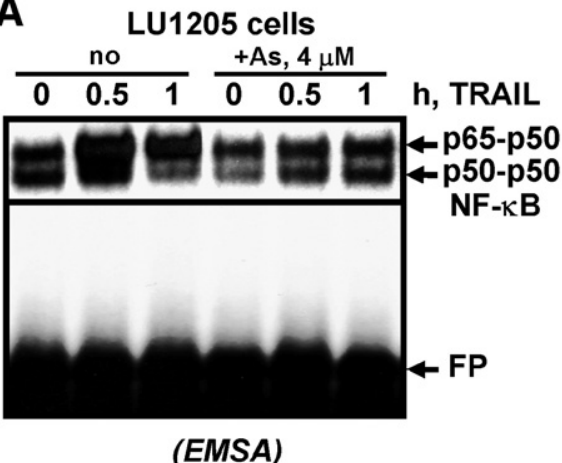

B

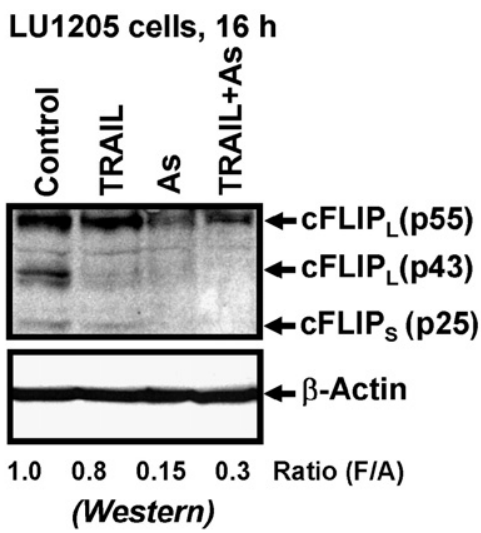

C

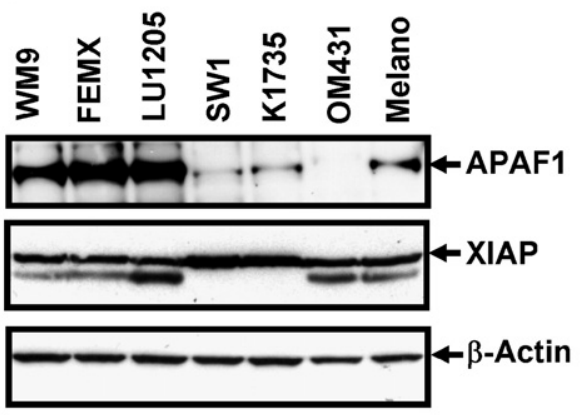

D

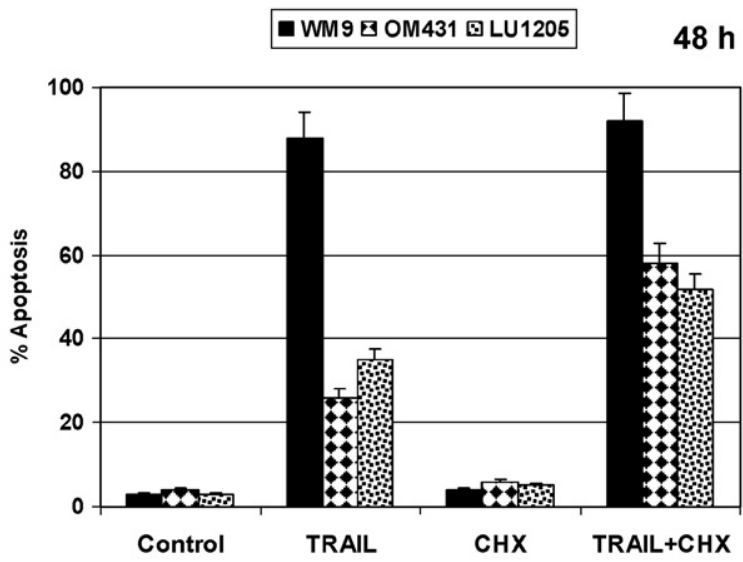

$\mathbf{E}$

口 WM9

$48 \mathrm{~h}$

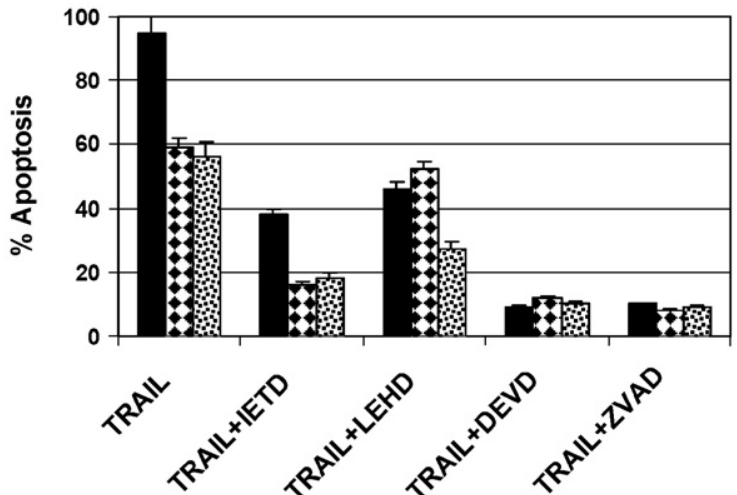

Fig. 2 - Modulation of TRAIL-mediated apoptosis in melanoma cells. (A) Effect of sodium arsenite pretreatment (4 $\mu \mathrm{M} ; 30 \mathrm{~min}$ ) on TRAIL-induced NF- $\kappa$ B activation (EMSA). The middle "empty" part of gel-shift has been removed. FP-free labeled probe. (B) Western blot analysis of cFLIP levels following treatments of LU1205 cells with TRAIL ( $50 \mathrm{ng} / \mathrm{ml})$ and sodium arsenite (4 $\mu \mathrm{M})$. A ratio CFLIP $_{\mathrm{L}}$ /Actin is indicated. Monoclonal Ab to FLIP (Dave-2) was used. (C) Western blot analysis of APAF1 and XIAP levels in melanoma cell lines. (D and E) Induction of apoptosis in indicated melanoma lines $48 \mathrm{~h}$ after indicated treatment. TRAIL $(50 \mathrm{ng} / \mathrm{ml})$ and CHX $(2 \mu \mathrm{g} / \mathrm{ml})$ have been used. Caspase inhibitors: IETD, LEHD, DEVD, zVAD ( $20 \mu \mathrm{M})$ have been added to the media $30 \mathrm{~min}$ before TRAIL + CHX. Cells were stained by PI $48 \mathrm{~h}$ after treatment. Apoptosis levels were determined as percentage of cells with hypodiploid content of DNA in the pre-G0/G1 region using flow cytometry. Error bars represent mean \pm SD from three independent experiments. 
Expression of TRAIL receptor-1 (TRAIL-R1/DR4) was detected on the surface of WM9 and OM431 human melanomas (Fig. 1A) and SW1 mouse melanoma cells (data not shown), in addition to TRAIL receptor-2 (TRAIL-R2/DR5) detected on the surface of LU1205 (0. 1A), WM9 and WM35 (data not shown) human melanomas. Hence, these cell lines were potentially susceptible to treatment with exogenous TRAIL. Indeed, soluble recombinant TRAIL (50 ng/ml) induced pronounced apoptosis of WM9 melanoma cells 24-48 h after treatment (Fig. 1B). This was the efficient caspase-8/caspase3-dependent apoptosis, which resulted in cleavage of PARP, a typical target of caspase-3, $6 \mathrm{~h}$ after treatment (Fig. 1C). However, effects of TRAIL on the induction of apoptosis in LU1205 and OM431 human melanomas and SW1 mouse cells, which contain modest levels of surface TRAIL-R, were less pronounced (Fig. 1B). Finally, FEMX human melanoma cells (R1-negative, R2-positive) only slightly responded to TRAIL (Figs. 1A, B).

Our next approach was to use a combined treatment of soluble TRAIL and sodium arsenite for possible acceleration of apoptosis in melanoma cells. Indeed, sodium arsenite $(4 \mu \mathrm{M})$ notably accelerated TRAIL-mediated apoptosis in WM9, OM431 and LU1205 melanoma cells $24 \mathrm{~h}$ after treatment (Fig. 1D). Finally, arsenite substantially increased levels of TRAILinduced apoptosis in OM431 and LU1205 human melanoma cells (Fig. 1E) and in mouse SW1 melanoma cells (data not shown) $48 \mathrm{~h}$ after treatment. At this time point, arsenite already had no additional effect on acceleration of apoptosis in TRAIL-sensitive WM9 cells.

Two critical features that may affect TRAIL-induced apoptosis in the presence of arsenite were (1) downregulation of basal and TRAIL-induced NF- $\kappa$ B activity and (2) downregulation of the $\mathrm{NF}-\kappa \mathrm{B}$-dependent targets, including cFLIP (both long and short forms). A general role of transcription factor NF- $\mathrm{BB}$ in protection against TRAIL-mediated apoptosis is well known $[39,40]$. Interestingly, soluble TRAIL $(50 \mathrm{ng} / \mathrm{ml})$ rapidly induced phospho-I $\mathrm{B} \alpha$ degradation in LU1205 melanoma cells (data not shown), which was accompanied by rapid induction of NF- $\kappa$ B DNA-binding activity. Pretreatment of LU1205 cell cultures with sodium arsenite (4 $\mu \mathrm{M}, 30 \mathrm{~min})$, which inhibited IKK $\beta$ activation [41], downregulated basal and TRAIL-induced IKK $\beta-N F-\kappa B$ activation (Fig. 2A). More pronounced inhibition of the basal NF- $\mathrm{B}$ activity by sodium arsenite could be achieved $6 \mathrm{~h}$ after treatment (see Figs. $3 \mathrm{C}$ and
$4 \mathrm{C})$. Since NF-кB controls expression of several genes encoding anti-apoptotic proteins, such as cFLIP, XIAP, cIAP1/2, TRAF1/2 and $\mathrm{BCl}-\mathrm{xL}$ [16], arsenite-induced downregulation of NF- $\mathrm{B}$ activity may partially suppress an anti-apoptotic response of cancer cells. As expected, we observed a downregulation of cFLIP expression (for three main isoforms) in LU1205 cells after both arsenite alone and a combined treatment of arsenite and TRAIL (Fig. 2B). Taken together, the effects of sodium arsenite (inhibition of NF- $\kappa$ B activity, downregulation of cFLIP protein levels and upregulation of surface TRAIL-R1/R2 levels) are consistent with the observed increase in the susceptibility of melanoma cells to TRAIL-mediated apoptosis (Figs. 1D and E).

Furthermore, TRAIL in combination with an inhibitor of protein synthesis, cycloheximide (CHX; $2 \mu \mathrm{g} / \mathrm{ml}$ ) (Fig. 2D), was more effective for an induction of apoptosis than TRAIL alone, probably due to a general inhibition of expression of shortlived anti-apoptotic proteins, including cFLIP [42]. Taken together, effects of sodium arsenite cotreatment were not synergistic, but additive to TRAIL-induced apoptosis, and were compatible with the final levels of TRAIL-induced apoptosis in the presence of CHX (Figs. 1E and 2D). As expected, zVAD-fmk $(5 \mu \mathrm{M})$, a universal caspase inhibitor, which was added into cell cultures $30 \mathrm{~min}$ before treatment, strongly downregulated levels of cell death, demonstrating a general dependence of TRAIL-mediated cell death on caspase activities in many melanoma lines, including WM9, OM431 and LU1205 cells (Fig. 2E and data not shown). Furthermore, TRAIL-induced apoptosis was efficiently suppressed by Ac-DEVD-CHO, a caspase-3 inhibitor. Ac-IETD-CHO $(5 \mu \mathrm{M})$, a caspase-8 inhibitor, and AC-LEHD-CHO $(5 \mu \mathrm{M})$, a caspase- 9 inhibitor, provided some protection against TRAIL-mediated apoptosis in WM9 and LU1205 cells. In contrast, Ac-LEHD-CHO was not effective in OM431 and SW1 cells with a deficiency in mitochondrial pathway, probably due to a strong downregulation of basal levels of apoptotic adaptor protein APAF1 (Figs. 2C and E). These data were well correlated with the involvement of both caspase-8 and caspase-9-mediated apoptotic pathways in the response to TRAIL in some melanoma cell lines. However, it also indicated that apoptotic death of melanoma cells with a deficiency in the mitochondrial pathway could be independent of caspase-9 activity. In conclusion, metastatic melanoma lines, WM9, LU1205, OM431 and SW1, responded to soluble TRAIL by induction of apoptosis that could be additionally increased with cotreatment either by $\mathrm{CHX}$ or by sodium

Fig. 3 - Dose-dependent effects of sodium arsenite for regulation of cell signaling and gene expression in WM9 melanoma cells. (A) Activation of ERK1/2, JNK, MAPK p38 and cJun $3 \mathrm{~h}$ after arsenite treatment (Western blot analysis). (B) Total and active form of STAT3, levels of TRAIL and TRAIL-R1 $6 \mathrm{~h}$ after arsenite treatment (Western). (C) Inhibition of NF- $\kappa$ B DNA-binding activity (EMSA) by sodium arsenite $6 \mathrm{~h}$ after treatment. Free labeled probe is not shown. (D) Western blot analysis of XIAP and cFLIP levels in WM9 cells $16 \mathrm{~h}$ after arsenite treatment. (E) NF- $\kappa B$-Luc, Jun2-Luc, GAS-Luc (STAT-dependent) reporter activities, the human TRAIL-promoter, TRAIL-R1-promoter and FLIP-promoter activities were determined $16 \mathrm{~h}$ after transient transfection of reporter constructs with an additional $(6 \mathrm{~h})$ exposure to indicated doses of arsenite. (F) Surface expression of TRAIL-R1 was determined before and $6 \mathrm{~h}$ after treatment of WM9 cells with arsenite (2-6 $\mu \mathrm{M})$. Cells were stained with PE-labeled mAbs and analyzed by the flow cytometry. Results of a typical experiment (one from three independent) are shown. (G) Surface expression of TRAIL was determined before and $6 \mathrm{~h}$ after treatment of WM9 cells with arsenite ( $4 \mu \mathrm{M})$. Cells were stained with PE-labeled mAbs and analyzed by the flow cytometry. Results of a typical experiment (one from three independent) are shown. (H) Suppression of arsenite-induced apoptosis by anti-TRAIL $\mathrm{mAb}(5 \mu \mathrm{g} / \mathrm{ml})$. Indicated mAbs were added into cell cultures $30 \mathrm{~min}$ before sodium arsenite. For apoptosis analysis, cells were stained by PI $48 \mathrm{~h}$ after treatment. Levels of apoptosis were determined as percentage of cells with hypodiploid content of DNA in the pre-G0/G1 region using flow cytometry. 
arsenite. In case of LOX and HHMSX melanomas, however, treatment by TRAIL in presence of CHX or arsenite still induced low levels of apoptosis (data not shown) indicating a need for different approaches for cell death induction.

Role of sodium arsenite treatment in the regulation of the endogenous TRAIL, TRAIL-R1/R2 expression and TRAIL-mediated apoptosis of melanoma cells

A potential role for soluble exogenous TRAIL as an inducer of apoptosis in sensitive melanoma lines is quite well established [11-13]. The next important questions are as follows: (1)
What is the mechanism of endogenous surface expression of TRAIL in TRAIL-R-positive melanomas that stimulates cell death via paracrine actions? (2) What is the mechanism by which sodium arsenite treatment affects levels of surface expression of TRAIL-R? (3) What is the mechanism by which sodium arsenite treatment affects endogenous CFLIP and XIAP expression in melanoma cells?

Three major points of downstream signaling that have profound effects on gene expression following sodium arsenite treatment of WM9 melanoma cells are as follows: (i) upregulation of the MAPK pathways [7,9] (Fig. 3A) that was accompanied by an increase in Jun2-Luc reporter activity (Fig.
A

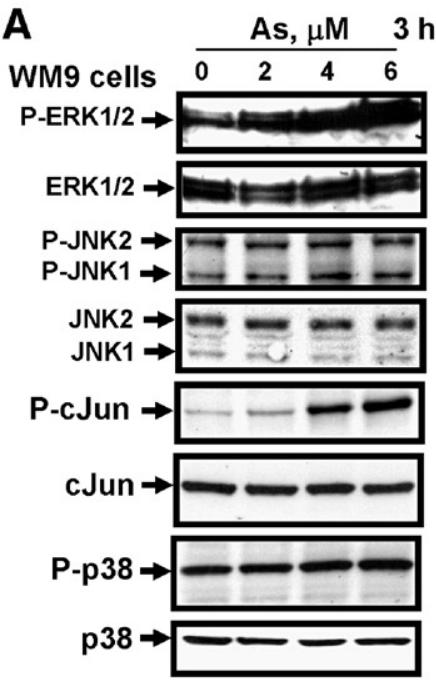

B

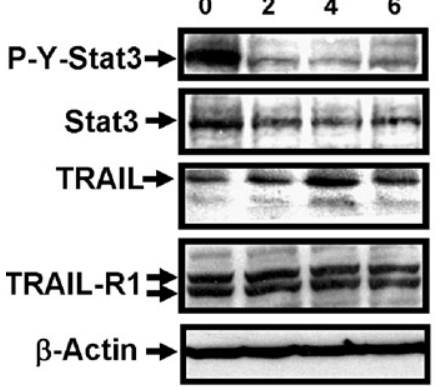

C

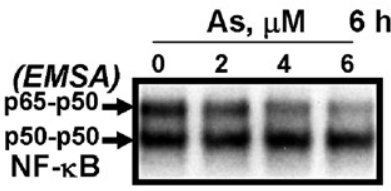

D

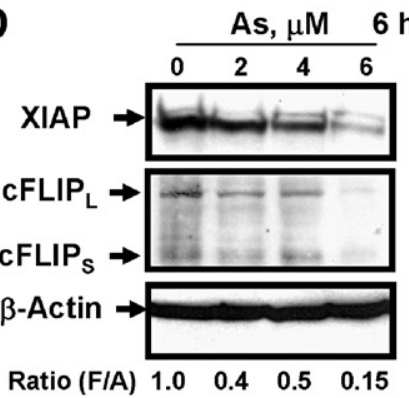

$\mathbf{E}$

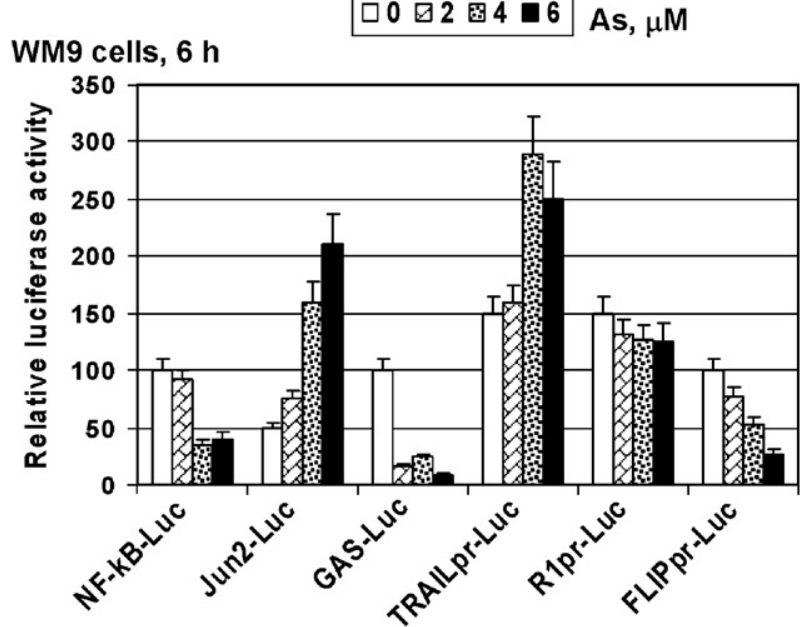

$\mathbf{F}$

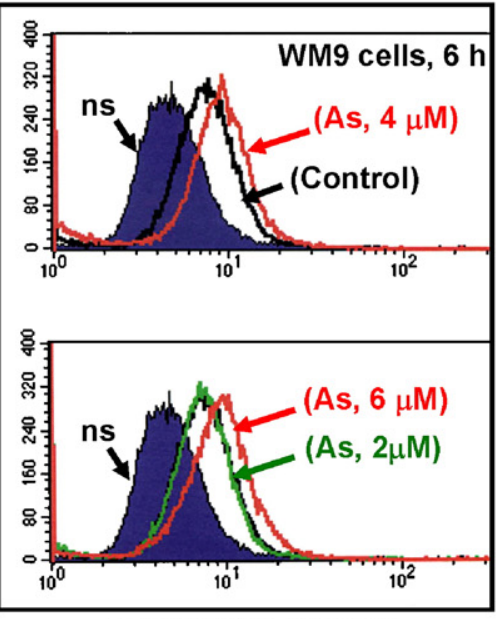

Surface TRAIL-R1 levels

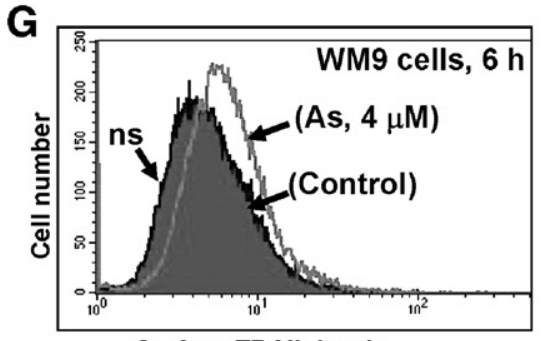

Surface TRAIL levels
H

WM9 cells, $48 \mathrm{~h}$

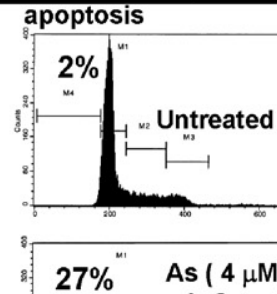
$+\lg G$

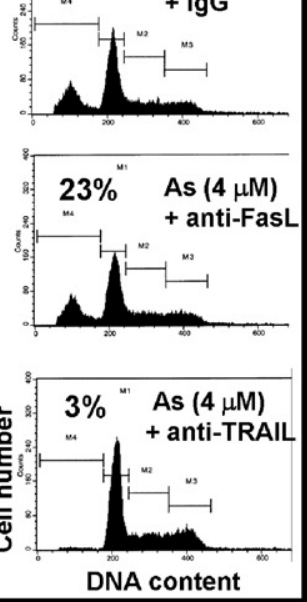


3E) and which may control TRAIL gene expression [43]; (ii) suppression of the basal IKK $\beta$ activity [41] and the basal NF- $\kappa B$ activity (Fig. 3C, EMSA; Fig. 3E, NF-кB-dependent transcription); and (iii) inhibition of JAK2 activity, which was detected by suppression of tyrosine phosphorylation of STAT3 (Fig. 3B) and resulted in downregulation of STAT3-dependent GAS-Luc reporter activity (Fig. 3E). Hence, arsenite treatment at low doses $(2-6 \mu \mathrm{M})$ was effective for downregulation of both NF- $\kappa B$ and STAT3 activities, while the MAPK/JNK-cJun pathway was substantially upregulated in WM9 melanoma cells in these conditions.

Previous studies on the transcriptional regulation of the endogenous TRAIL gene have demonstrated its control by NF$\kappa B$ and STAT3 in concert with several other transcription factors, including SP1, AP1 and GATA [26,43]. Intracellular TRAIL protein was easily detectable in WM9 melanoma cells (Fig. 3B). However, TRAIL was not present on the cell surface of nontreated melanoma cells (Fig. 3G). Determination of the TRAIL promoter reporter activity (with high basal level in WM9 cells) demonstrated a relatively modest upregulation of this activity $6 \mathrm{~h}$ after arsenite treatment $(4 \mu \mathrm{M})$ (Fig. 3E). Furthermore, we observed the anticipated effects of NF- $\kappa$ B, AP1 and STAT3 in the regulation of the TRAIL promoter activity in melanomas. Super-stable I $\mathrm{B} \alpha \Delta \mathrm{N}$, which was a very effective inhibitor of NF-kB, had a relatively small negative effect on the TRAIL promoter. In contrast, pronounced inhibitory effects of dominant-negative STAT3-F, JNK1-APF and TAM67 (dominant negative form of cJun) have been observed. On the other hand, permanently active variant, STAT3-C, was a powerful activator of the TRAIL promoter, as well as an active JNKK (MKK7- $\beta 1$ ) that was upstream of JNK. Simultaneously, permanently active AKTmyr, which suppressed both JNK-cJun and STAT3 activation, also strongly suppressed the TRAIL promoter activity (data not shown). These experimental data confirmed that STAT3 and AP1/CJun are the main positive regulators of the TRAIL transcription, while a role of NF- $\mathrm{kB}$ in this regulation was relatively minor for melanoma cells.

Hence, due to strong activation of cJun (via MAPK/JNK), in spite of the downregulation of STAT3 and NF- $\kappa$ B activities, arsenite treatment actually increased the total TRAIL promoter activity and TRAIL protein levels (Figs. 3B and E). Activating effects of arsenite on TRAIL promoter and TRAIL protein level were maximal at the dose $4 \mu \mathrm{M}$ for WM9 cells (Fig. 3B). Furthermore, arsenite (but not Bay7082, a standard IKK-NF-кB suppressor) induced TRAIL expression on the cell surface, indicating that this function was independent of NF-кB inhibition (Fig. 3G). Arsenite treatment also upregulated surface expression of TRAIL-R1/R2 in several melanoma lines, including WM9 (Fig. 3F), in spite of the fact that TRAIL-R1 gene promoter activity and total protein levels of TRAIL-R1 were relatively stable in these conditions (Figs. 3B and E). These results further suggested an effect of sodium arsenite on the regulation of TRAIL-R1 protein trafficking to the cell surface. Upregulation of TRAIL-R surface levels by arsenite treatment was previously observed for multiple myelomas [44] and some leukemic cell lines [45].

Arsenite treatment of WM9 melanoma cells resulted in a modest upregulation of both TRAIL and TRAIL-R1 surface levels. Furthermore, a dramatic dose-dependent downregulation of the cFLIP promoter activity and expression, as well as downregulation of expression of XIAP, were also observed $16 \mathrm{~h}$ after arsenite treatment (Figs. 3D, E). Importantly, expression of both CFLIP and XIAP genes is known to be NF- $\kappa$ B dependent [46]. Taken together, these arsenite-induced changes may lead to induction of apoptosis. Indeed, dose-response effects of sodium arsenite on induction of caspase-3-dependent apoptosis were revealed in WM9 cells that could be suppressed by anti-TRAIL mAb in cell culture (Fig. $3 \mathrm{H}$ and data not shown). PARP cleavage (which could also be blocked with anti-TRAIL $\mathrm{mAb}$ ) served as an additional proof of caspase-3-dependent mechanism of apoptosis in WM9 cells following indicated treatments (data not shown). In summary, our data indicated that for some melanoma lines (WM9, LU1205, SW1), arsenite treatment by itself induced a modest TRAIL-mediated apoptosis $48 \mathrm{~h}$ after treatment based on surface expression of TRAIL and TRAIL-R and paracrine induction of cell death. However, it is more important to note that arsenite may also effectively increase the susceptibility of melanoma cells to exogenous TRAIL due to upregulation of surface TRAIL-R levels and downregulation of cFLIP and XIAP.

WM9 metastatic melanoma cells are highly sensitive to exogenous TRAIL, while LU1205 metastatic melanoma cells are relatively resistant and require sodium arsenite or $\mathrm{CHX}$ cotreatment to overcome this resistance. Nevertheless, many effects of arsenite treatment on cell signaling pathways were substantially similar in both cell lines: upregulation of the MAPK/JNK-cJun pathway, downregulation of STAT3 and NF-кB activation, which was accompanied by downregulation of cFLIP levels (Figs. 3 and 4). Arsenite treatment also upregulated TRAIL promoter activity, TRAIL protein levels and TRAIL surface expression and increased levels of surface TRAIL-R2 in LU1205 cells (Figs. 4A, D and E). However, at least two critical parameters are different in LU1205 and WM9 cells: higher basal levels of active AKT, due to PTEN dysfunction, and higher levels of basal NF- $\mathrm{kB}$ activity in LU1205, compared to WM9 cells $[47,48]$. These differences are well correlated with substantially higher levels of the cFLIP promoter activity and cFLIP protein expression in LU1205 cells (Figs. 4F and G), which may explain their relative resistance to TRAIL.

\section{Regulation of the cFLIP promoter activity and cFLIP expression in} melanoma cells

Expression of cFLIP and XIAP, which was known to be NF- $\kappa$ B dependent $[16,42,49]$, has been dramatically suppressed by arsenite treatment (Figs. 3E, D and 4B, D). In order to more precisely characterize the regulation of cFLIP/CFLAR expression, we used reporter constructs containing the human cFLIP promoter $[28,29]$. In an addition to previously described ciselements, such as SP1- and cMyc-binding sites, this promoter contains four putative AP1-binding sites, one putative CRE site and two GAS sites that could bind STAT3, but no NF-кBbinding sites (Fig. 5A). Using transient transfection of hFLIPprLuc together with different expression constructs encoding dominant-negative and permanently active forms of transcription factors and corresponding kinases, we observed dramatic activating effects of cJun-dominant-negative form (TAM67) and strong inhibitory effects of active MEKK1 (as MAP3K) and MKK7- $\beta 1$ (as JNKK) on hFLIP promoter activity (Fig. 5B). The effect of TAM67 on upregulation of protein levels of cFLIP was relatively transient and was quickly followed by 
A

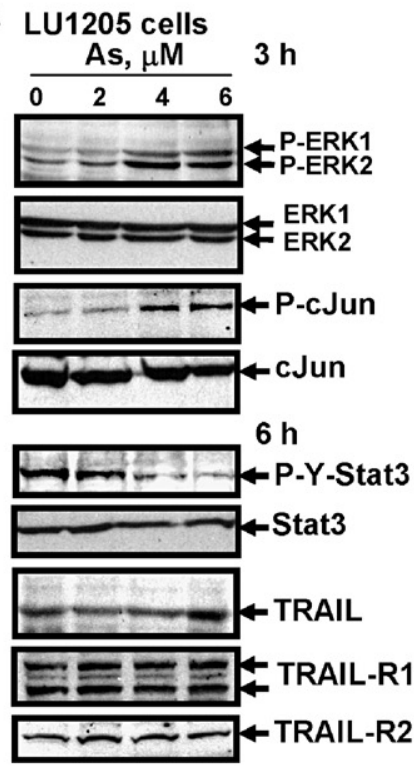

B

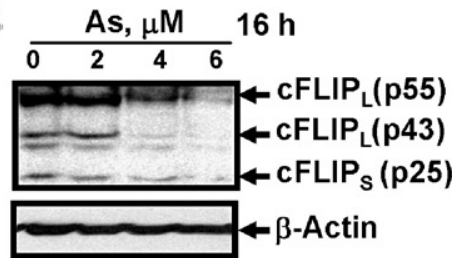

$\begin{array}{lllll}1.0 & 0.7 & 0.3 & 0.1 & \text { Ratio (F/A) }\end{array}$

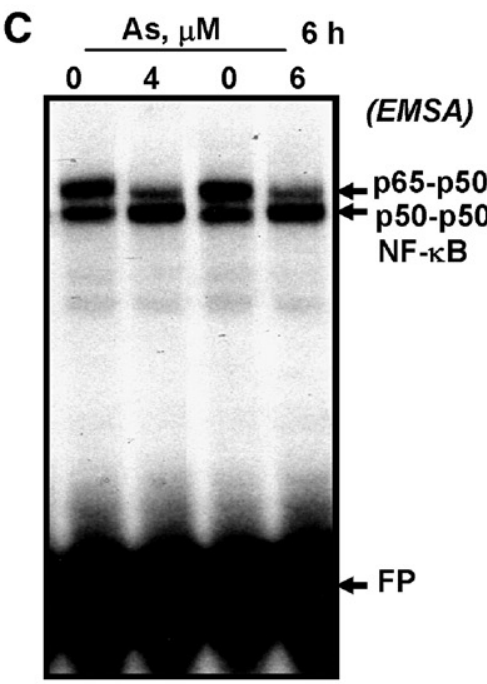

D

LU1205 cells, $6 \mathrm{~h}$

TRAILpr-Luc $\mathbf{\Delta}$ hFLIPpr-Luc

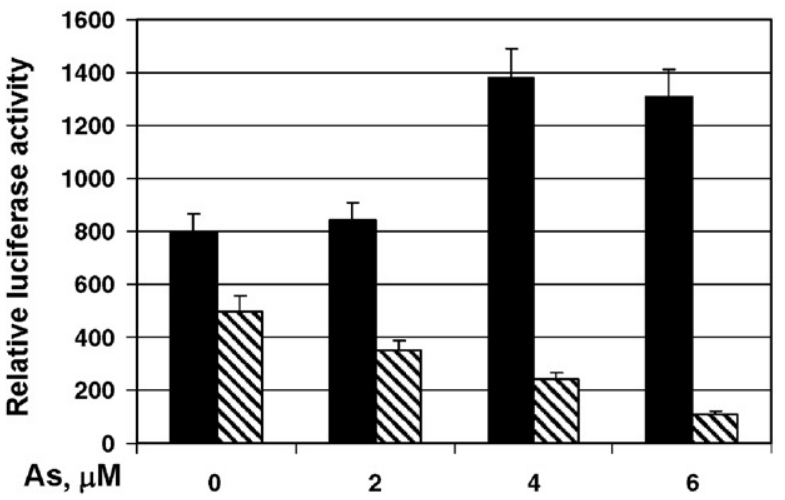

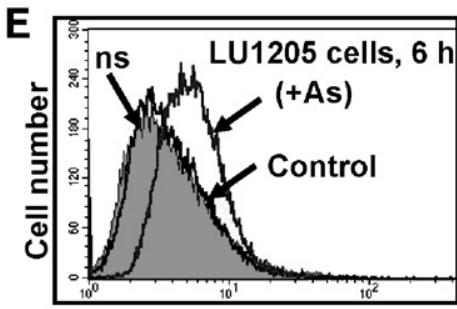

Surface TRAIL levels

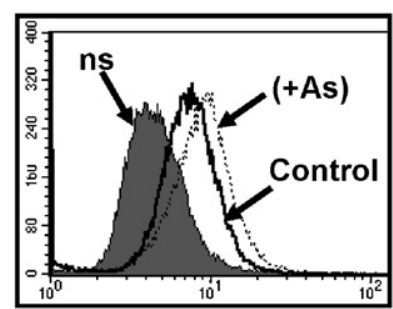

Surface TRAIL-R2 levels F

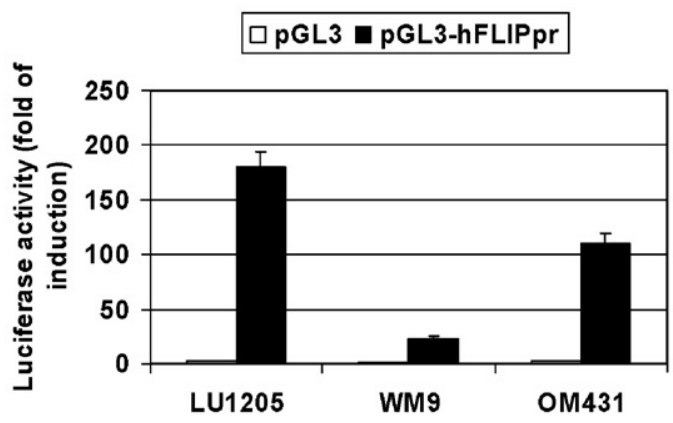

G

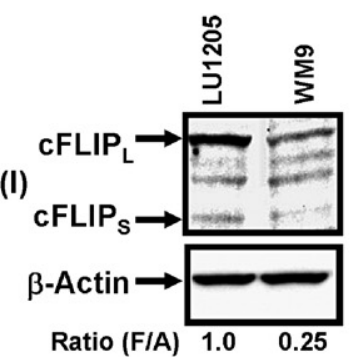

Fig. 4 - Regulation of cell signaling and gene expression by sodium arsenite in LU1205 melanoma cells. (A) Activation of ERK1/2 and cJun $3 \mathrm{~h}$ after arsenite treatment (Western blot analysis). Inhibition of STAT3 activity, levels of TRAIL and TRAIL-R1 and R2 $6 \mathrm{~h}$ after arsenite treatment (Western). (B) Western blot analysis of cFLIP levels $16 \mathrm{~h}$ after arsenite treatment. Dave-2 mAb to FLIP was used. (C) Inhibition of NF- $\kappa$ B DNA-binding activity (EMSA) by sodium arsenite $6 \mathrm{~h}$ after treatment. (D) The human TRAIL- and FLIP-promoter activities were determined $16 \mathrm{~h}$ after transient transfection of reporter constructs with an additional $(6 \mathrm{~h})$ exposure to indicated doses of arsenite. (E) Surface expression of TRAIL and TRAIL-R2 was determined before and $6 \mathrm{~h}$ after treatment of LU1205 cells with arsenite ( $4 \mu \mathrm{M})$. Cells were stained with correspondent PE-labeled mAbs and analyzed by the flow cytometry. Results of a typical experiment (one from three independent) are shown. (F) Normalized basal levels of the hFLIP promoter activities in indicated melanoma lines; pGL3-basic-the empty vector. Error bars represent mean \pm SD from three independent experiments. $(G)$ The basal protein levels of cFLIP in indicated melanoma lines determined by Western blot analysis. Monoclonal Ab to FLIP (Dave-2) was used. 


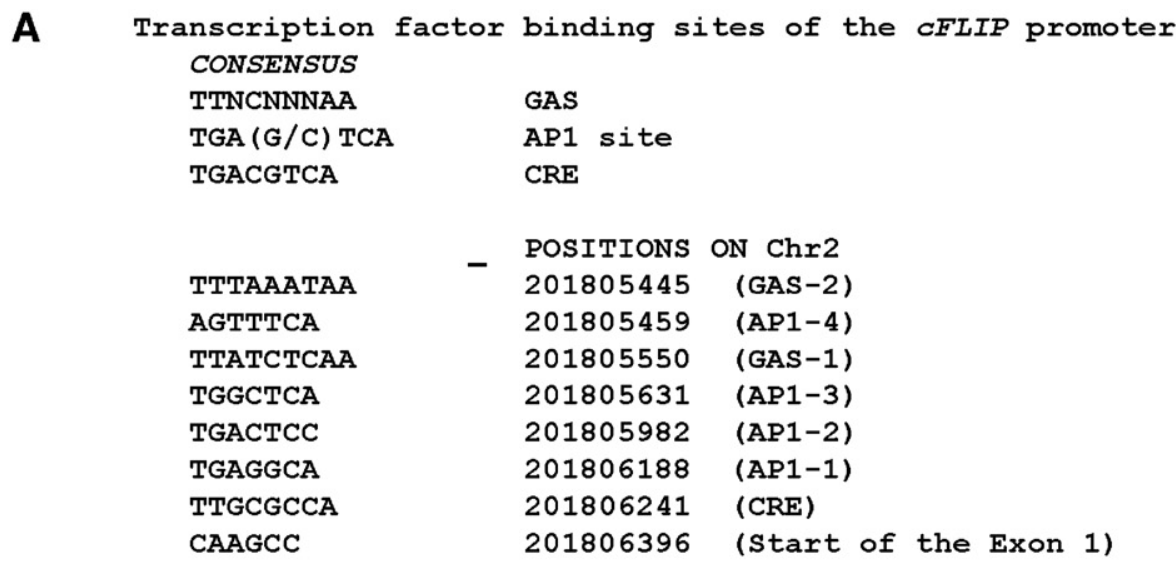

B

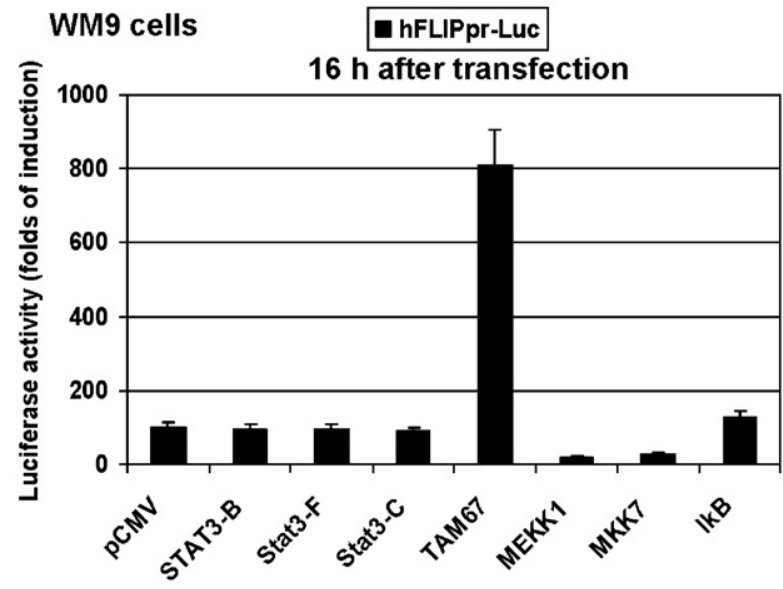

C

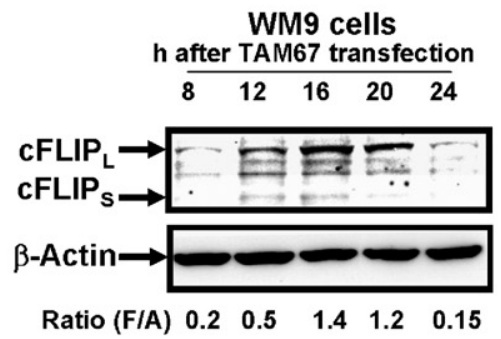

D WM9 cells

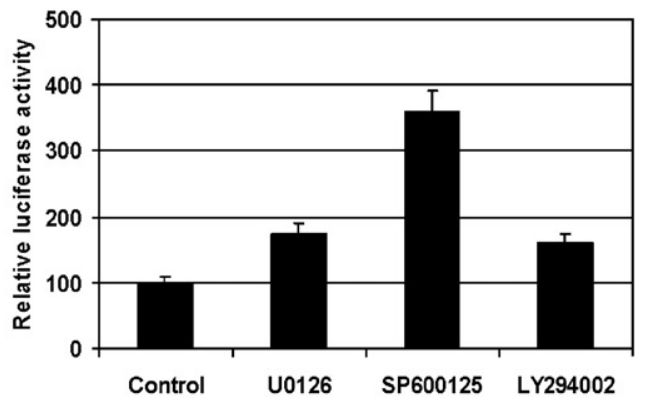

E

\section{LU1205 cells ahFLIPpr-Luc DhFLIPpr-LuctAs}

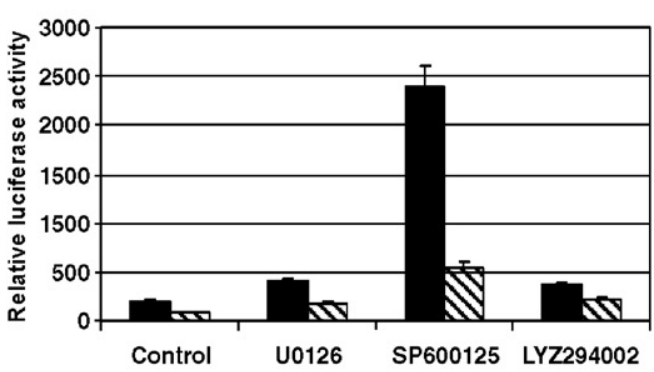

Fig. 5 - The cFLIP promoter analysis. (A) Putative transcription factor-binding sites of the human cFLIP/CFLAR gene promoter. Position of binding sites on the human chromosome-2 is indicated. (B) Transient transfection of hFLIPpr-Luc and expression vectors encoding indicated dominant-negative and permanently active forms of proteins in the presence of $\beta$-gal expression construct (at a plasmid DNA ratio 1:3:0.5) into WM9 cells. Normalized luciferase activity was determined $16 \mathrm{~h}$ after

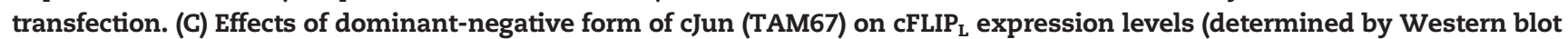
analysis) after transient transfection of WM9 cells. (D, E) Effects of specific inhibitors: of the MEK-ERK pathway, U0126 (10 $\mu$ M), of JNK, SP600125 (20 $\mu \mathrm{M})$, of the PI3K-AKT pathway, LY294002 (20 $\mu \mathrm{M})$ on hFLIPpr-Luc activity in transiently transfected WM9 and LU1205 melanoma cells. Arsenite (As, $4 \mu \mathrm{M}$ ) was used. Cells were treated for $6 \mathrm{~h}$ before determination of normalized luciferase activity.

downregulation of cFLIP expression to basal levels (Fig. 5C). The effects of TAM67 were well correlated with a strong increase in cFLIP promoter activity after inhibition of JNK activation by specific pharmacological inhibitor SP600125
(Figs. 5D and E), while the presence of arsenite dramatically diminished this effect. The effect of U0126, a MEK-ERK inhibitor, was less pronounced in both WM9 and LU1205 cells. In contrast, STAT3 expression constructs (both active 
STAT3-C and dominant-negative STAT3-F and STAT3 $\beta$ ) as well as super-stable I $\mathrm{B} \Delta \mathrm{N}$ had no real effects on the FLIP promoter (Fig. 5B). However, since FLIP protein levels are known to be NF- $\kappa$ B-dependent based on several studies $[42,49]$, it probably indicated an indirect relationship, which could be mediated by the product of another NF- $\kappa$ B-regulated gene. In this regard, the COX-2 gene that is permanently active in melanomas [48] and is under direct control of NF- $\mathrm{BB}$ [50] is a possible candidate. A detailed characterization of human FLIP promoter and regulation of CFLIP gene expression are still in progress. In summary, the negative effects of arsenite treatment on CFLIP promoter activity are mediated by MAPK/JNK-cJun pathways. Interestingly, a recent study has described a JNK-mediated protein degradation of cFLIP via activation of the E3 protein ligase Itch [51], confirming the important role of MAPK/JNK in the regulation of FLIP expression at the different control points.

In order to further confirm a critical role of cFLIP downregulation for accelerating TRAIL-mediated apoptosis in human melanomas, we created several cFLIP RNAi constructs (based on Oligoengine-developed strategy), FLIP-92 RNAi construct effectively suppressed CFLIP $_{\mathrm{L}}$ and $\mathrm{CFLIP}_{\mathrm{S}}$ expression in LU1205 (Fig. 6A), OM431 and SW1 melanomas (data not shown). We used two different mAbs to human cFLIP: Dave-2 (I) and NF6 (II) (Fig. 6A). Both mAbs detected downregulation of CFLIP $_{\mathrm{L}}$ expression following RNAi-mediated suppression; furthermore, NF6 mAb also detected a decrease in cFLIP $_{\mathrm{s}}$ levels, while Dave- $2 \mathrm{mAb}$ was not able to efficiently detect the short form of FLIP neither in control cells nor in FLIP-RNAitransfected cells after puromycin-based selection. RNAimediated reduction of cFLIP expression substantially upregulated TRAIL- or (TRAIL and CHX)-induced apoptosis of control LU1205, OM431 and SW1 cells but did not affect levels of arsenite-induced apoptosis because arsenite by itself was involved in the inhibition of cFLIP (Figs. 6B, C and data not shown).

COX-2 suppression downregulates cFLIP levels and accelerates TRAIL- and FasL-mediated apoptosis in human melanomas The COX-2-cFLIP-positive axis was recently described for some cell systems [52] and it is likely to be true in melanomas as well, where both COX-2 and cFLIP are permanently active. We suppressed COX-2 expression in LU1205 and WM9 cell lines using several COX-2 RNAi expressing constructs: COX-2-379 RNAi was the most effective. It was also accompanied by a pronounced suppression of both $\mathrm{CFLIP}_{\mathrm{L}}$ and $\mathrm{CFLIP}_{\mathrm{S}}$ (Fig. 6D). Effects of the COX-2prostaglandin-E2 signaling pathway on CFLIP expression were mediated via upregulation of the cFLIP promoter activity since suppression of COX-2 downregulated the CFLIP promoter activity (Fig. 6E). In contrast, we did not observe pronounced effects of COX-2 suppression on TRAIL promoter activity (Fig. 6E) or total TRAIL levels (data not shown). In summary, COX-2 suppression notably increased levels of TRAIL- or (TRAIL+CHX)-induced apoptosis in LU1205 cells (Fig. 6F). The inhibitory effects of COX-2 on JNK-cJun activities [53] may be involved in downregulation of cFLIP levels following suppression of COX-2 by specific RNAi.

Furthermore, COX-2 suppression by specific RNAi decreased levels of surface expression of TRAIL after arsenite treatment of LU1205 melanoma cells (Figs. 7A and B), indicating a possible role of COX-2 in the regulation of TRAIL protein translocation to the cell surface. Unexpectedly, the effect of COX-2 suppression combined with arsenite on TRAIL surface expression was the reverse to the previously observed upregulation of FasL surface expression in these conditions [48]. However, arsenite-induced apoptosis of melanoma cells with suppressed COX-2 was dramatically increased. Pretreatment with monoclonal antibodies against FasL, TRAIL or TNF $\alpha$ demonstrated that this increase was mainly due to FasLmediated death signaling; however, TRAIL-mediated death was still involved (Fig. 7C). Taken together, COX-2 downregulation was determined as a powerful tool for upregulation of arsenite-induced apoptosis of human melanoma through both FasL-Fas and TRAIL-TRAIL-R pathways with simultaneous cFLIP suppression.

Downregulation of TRAIL-R surface expression by AKT suppresses TRAIL-mediated apoptosis in melanoma The PI3K-AKT pathway, which controls and coordinates many survival functions in the cell (including high basal COX-2 levels), may suppress arsenite-induced sensitization to TRAILmediated apoptosis [54]. In contrast, suppression of the PI3KAKT pathway by specific inhibitor LY294002 may additionally increase levels of (TRAIL $+\mathrm{CHX}$ )-induced apoptosis of human melanoma cells (Fig. 7E). The total effects of LY294002 (50 $\mu \mathrm{M})$ cotreatment on TRAIL-induced cell death were even more pronounced due to relatively high levels of necrotic death in treated melanoma cells in these conditions (Fig. 7E and data not shown). High levels of the secondary necrotic cells (PI-positive, Annexin-V-negative) have been observed even $12 \mathrm{~h}$ after TRAIL treatment in the presence of LY294002 (Fig. 7D). TRAIL-induced death of mouse metastatic melanoma cells was also substantially increased in the presence of LY294002 (see Fig. 8C).

In these experiments, we used previously established mouse SW1 melanoma cells stably transfected either with the empty vector or with the construct expressing permanently active AKTmyr [55]. Overexpression of active AKT caused downregulation of AP1- and STAT3-dependent transcription and modest upregulation of NF- $\kappa$ B activity and NF$\kappa \mathrm{B}$-dependent transcription (Figs. 8A, B and data not shown). The general transcriptional changes induced by AKT overactivation resulted in upregulation of the Fas promoter activity [55], while the FasL promoter activity [56], as well as the TRAIL promoter and TRAIL-R1 promoter activities, was notably downregulated (Fig. 3F and data not shown). Correspondingly, Western blot analysis demonstrated a strong decrease in endogenous protein level of TRAIL, FasL and modest decrease in total TRAIL-R1 protein levels in AKTmyr-transfected cells (Fig. 8A). Furthermore, surface expression of TRAIL-R1 was substantially decreased in AKTmyr-transfected cells, even less in the presence of arsenite, revealing a critical level of decreased sensitivity to TRAIL in these cells (Fig. 8D). Indeed, there was a substantially downregulated sensitivity to apoptotic signaling induced by soluble exogenous TRAIL or by a combination of TRAIL and CHX in AKT-transfected melanoma cells (Fig. 8C). This was in the contrast to increased sensitivity to exogenous FasL, which was previously observed in these cells [55]. AKT-mediated upregulation of COX-2 levels and 
A

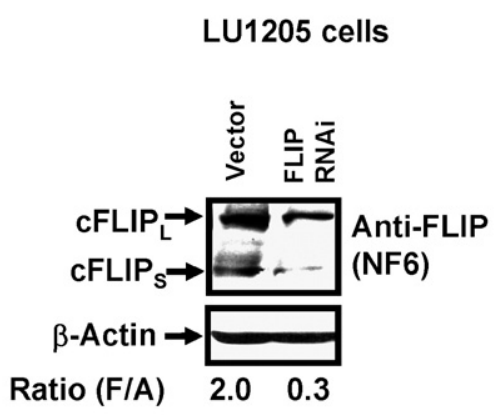

B

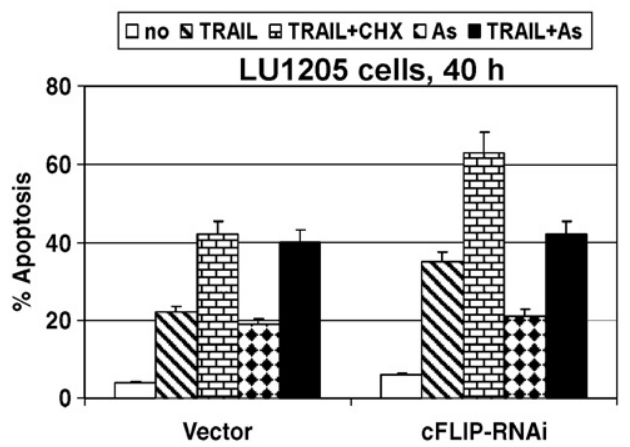

C

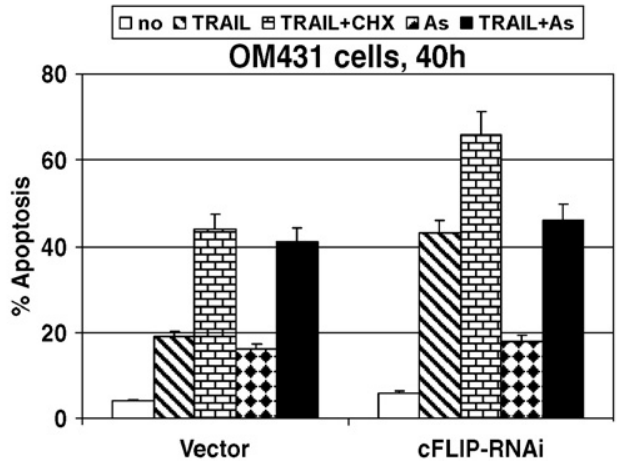

D

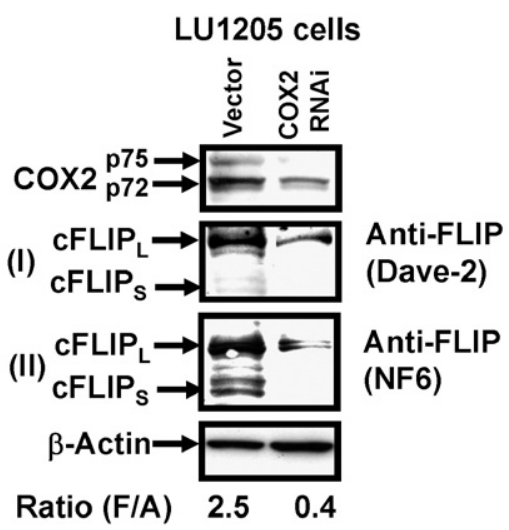

E

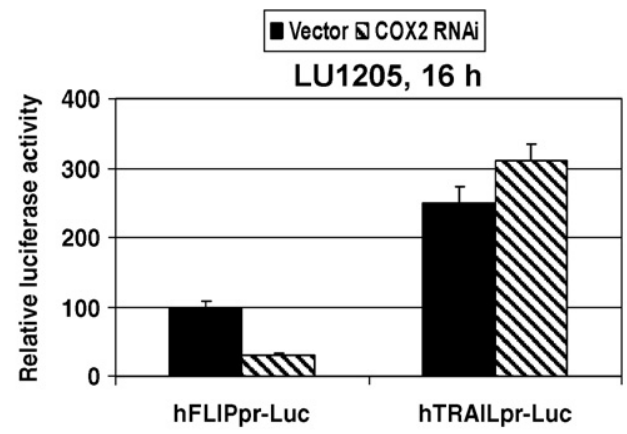

$\mathbf{F}$

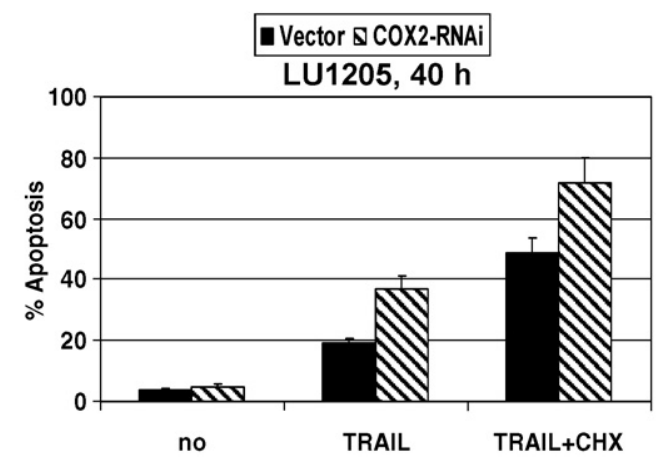

Fig. 6 - Suppression of cFLIP expression by cFLIP-RNAi or COX2-RNAi accelerates TRAIL-mediated apoptosis of melanoma cells. (A) Western blot analysis of cFLIP levels in LU1205 cells stably transfected with the empty vector pSR-puro or with RNAi cFLIP-92. Long and short form of cFLIP are indicated. Monoclonal Ab to FLIP (clone NF6) (II) was used. (B, C) Levels of apoptosis were determined $40 \mathrm{~h}$ after indicated treatment of LU1205 and OM431 cells stably transfected with the empty vector or RNAi cFLIP-92. Error bars represent mean \pm SD from three independent experiments. (D) Suppression of COX-2 expression by COX-2 RNAi downregulates cFLIP levels. Western blot analysis of COX-2 and cFLIP levels (with two types of mAbs against cFLIP, Dave-2 and NF6) in LU1205 cells stably transfected with the empty vector pSR-puro or RNAi COX2-379. Two main protein bands of COX2 (p72 and glycosylated form p75) are indicated. (E) Effects of RNAi COX2-379 on hFLIP- and hTRAIL-promoter activities. LU1205 cells were transiently transfected with hFLIP-Luc, pSR-puro and $\beta$-gal (1:3:0.5) or with hFLIP-Luc, RNAi COX-2 and $\beta$-gal (1:3:0.5). Normalized Luc activity was determined $16 \mathrm{~h}$ after transient transfection.

(F) Apoptosis levels in LU1205 cells stably transfected with the empty vector pSR-puro or RNAi COX2-379 $40 \mathrm{~h}$ after treatment with soluble TRAIL $(50 \mathrm{ng} / \mathrm{ml})$ or TRAIL + CHX $(2 \mu \mathrm{g} / \mathrm{ml})$.

modest upregulation of the basal NF-кB levels might provide additional survival signaling in these cells (Figs. 8A and B).

Taken together, our results confirmed a protective role of AKT against TRAIL-induced apoptosis of melanoma in cell culture conditions. The next question to be addressed was melanoma growth in vivo after injection of the control (transfected by the empty vector) SW1 melanoma cells and AKTmyr-transfected SW1 cells into its natural host, $\mathrm{C} 3 \mathrm{H} /$ 


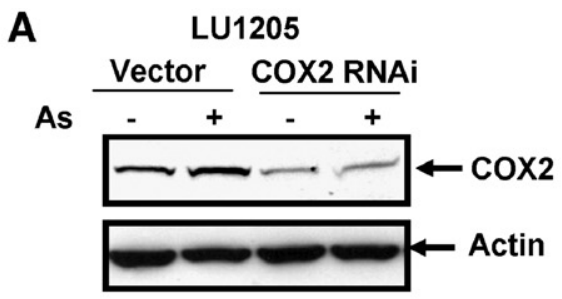

B

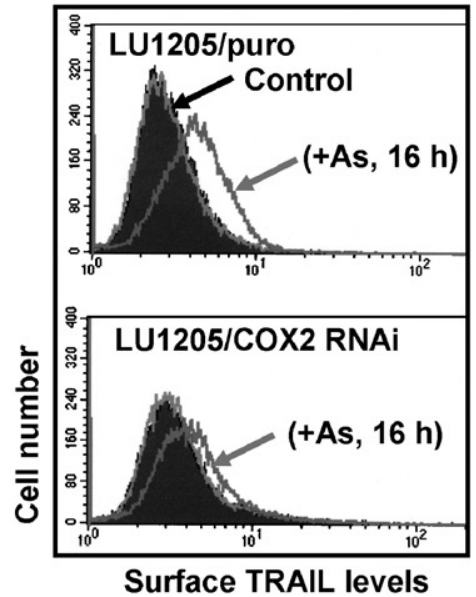

D

Control
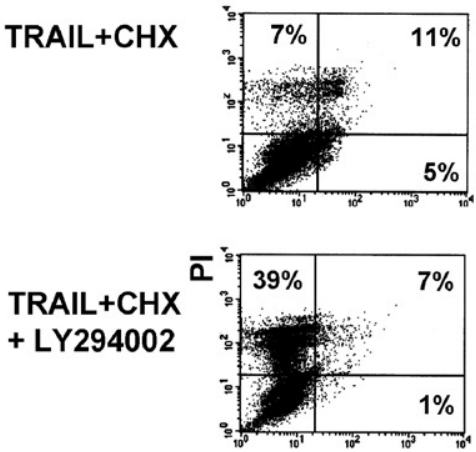

Annexin-V-FITC

C

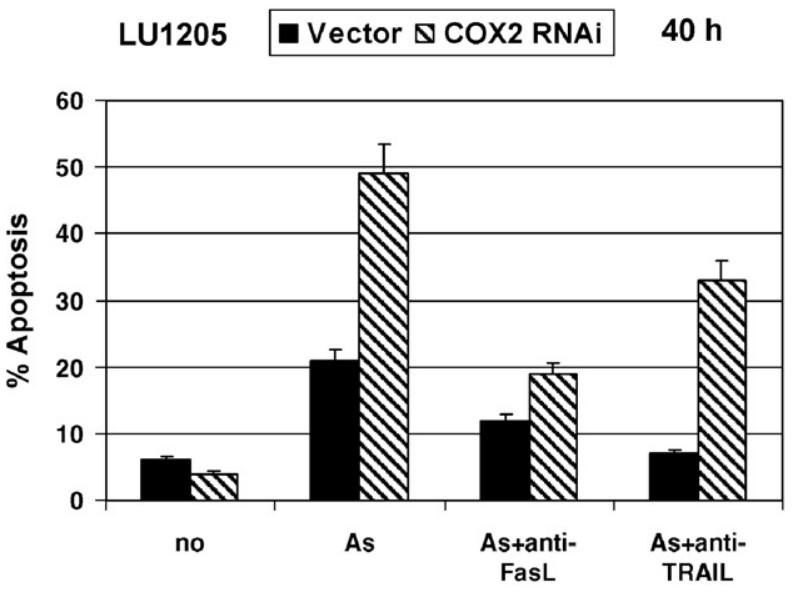

E

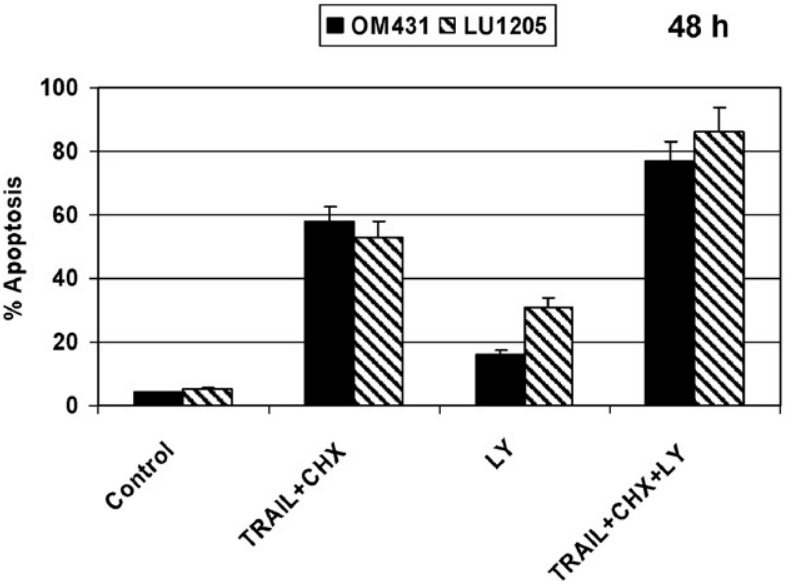

Fig. 7 - Effects of COX-2 and PI3K-AKT suppression on arsenite- and TRAIL-induced apoptosis in melanoma cells. (A) LU1205 cells stably transfected with the pSR-puro or with RNAi COX2-379 were treated for $6 \mathrm{~h}$ with arsenite (4 $\mu \mathrm{M})$. COX-2 protein levels were detected by Western blot analysis. (B) Surface TRAIL levels in LU1205-puro and LU1205/COX-2 RNAi stably transfected cells before (control) and after arsenite treatment. (C) Effects of pretreatment with anti-FasL or anti-TRAIL $\mathrm{mAb}(5 \mu \mathrm{g} / \mathrm{ml})$ on apoptosis induced by arsenite $(4 \mu \mathrm{M})$ treatment. Error bars represent mean \pm SD from three independent experiments.

(D) LU1205 cells were stained by Annexin-V-FITC and PI $12 \mathrm{~h}$ after indicated treatment. (E) LU1205 and OM431 cells were treated by TRAIL + CHX $48 \mathrm{~h}$ with or without LY294002 (LY, $50 \mu \mathrm{M})$. Apoptosis levels were determined as percentage of cells with hypodiploid content of DNA in the pre-G0/G1 region using flow cytometry. Error bars represent mean \pm SD from three independent experiments.

HEJ mice. Previous investigations with enhancement of apoptotic responses of SW1 cells revealed a substantial downregulation of tumor growth [57]. In contrast, the effect of AKT overexpression in melanoma was a dramatic acceleration of tumor growth in mice (Figs. 8E-G). This was likely the result of AKT-mediated positive control of 


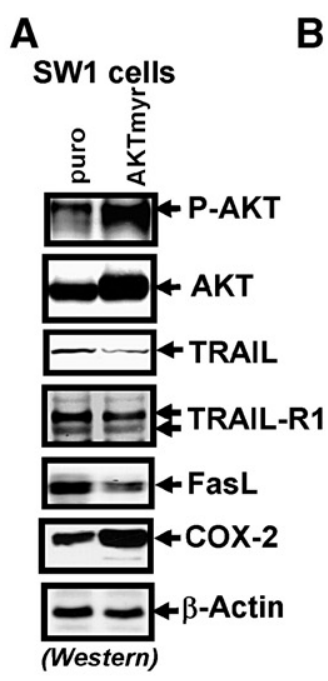

D

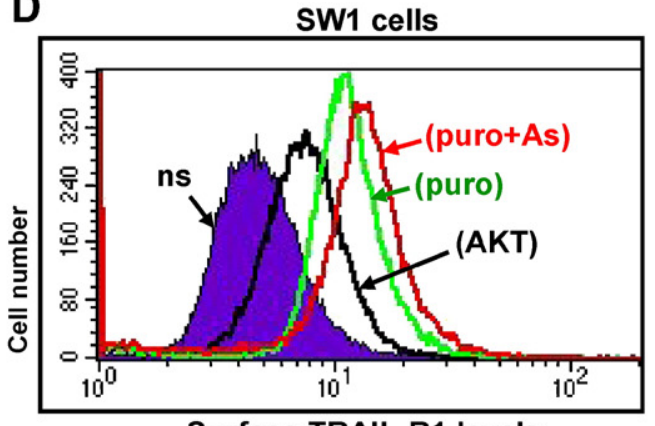

Surface TRAIL-R1 levels

$\mathbf{E}$

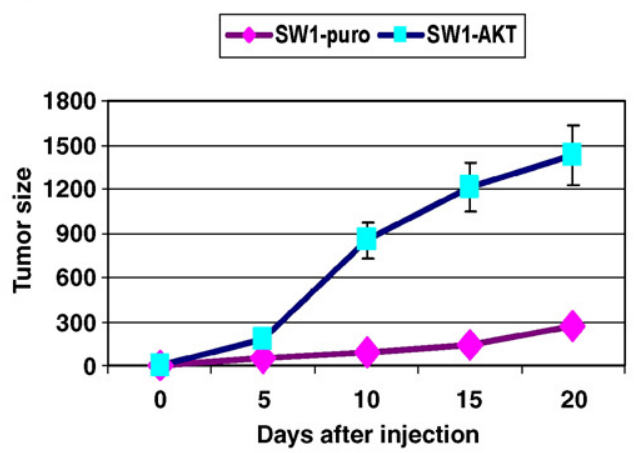

C

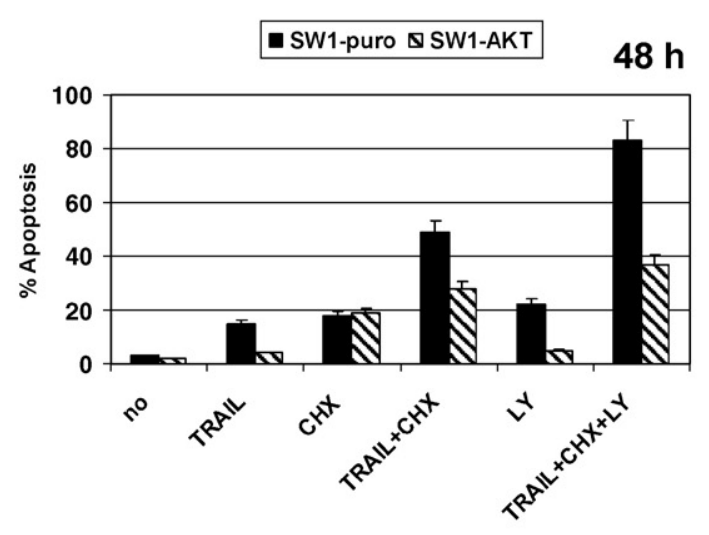

$\mathbf{F}$

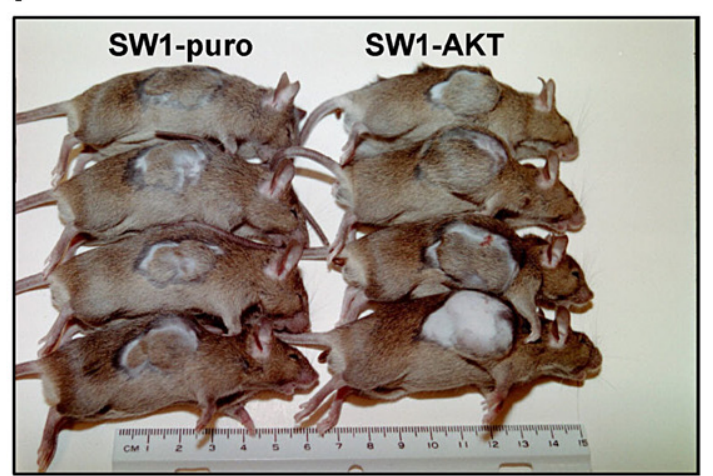

G

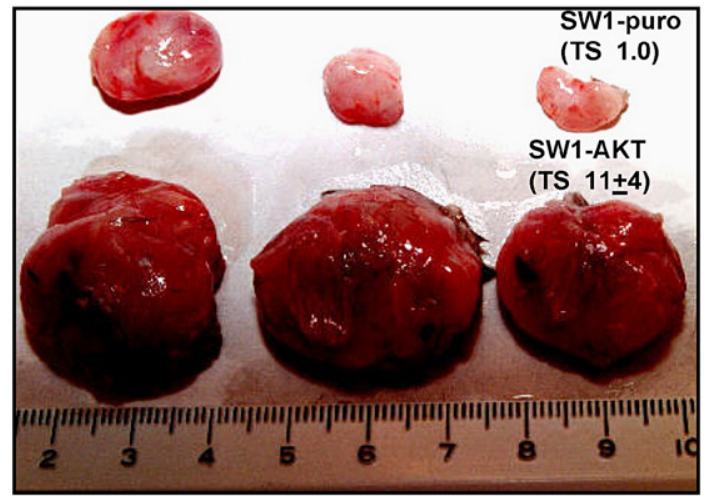

Fig. 8 - AKTmyr overexpression downregulates TRAIL-mediated apoptosis of SW1 melanoma cells and dramatically accelerates SW1 melanoma transplant growth in vivo. (A) Western blot analysis of indicated proteins in SW1 melanoma cells stably transfected with the empty vector or AKTmyr expression construct. (B) Effects of AKTmyr on NF- $\kappa B$ DNA-binding activity determined by EMSA. (C) Overexpression of AKTmyr downregulated TRAIL-, (TRAIL + CHX)- and (TRAIL+CHX+LY)-induced apoptosis of SW1 cells . (D) Surface TRAIL-R1 levels in SW1 cells stably transfected with the empty vector (puro) or AKTmyr expression construct before and after arsenite treatment. Cell were stained with anti-TRAIL-R1 mAb and analyzed by the flow cytometry. Results of typical experiment (one from three independent) are shown. (E-G) SW1 melanoma cells, permanently transfected with the empty vector or with AKTmyr expression construct, were injected subcutaneously into C3H/HEJ mice (ten mice for each group), and tumor growth was monitored during the next three weeks. TS-relative tumor size.

cancer cell survival functions, including a partial suppression of TRAIL-mediated signaling due to downregulation of TRAIL-R1 surface expression (Fig. 8D). This protects mela- nomas from targeting by TRAIL-producing natural killer cells and cytotoxic $\mathrm{T}$ lymphocytes, thus facilitating tumor development. 


\section{Discussion}

Arsenic (as arsenic trioxide or sodium arsenite) is a potent inducer of apoptosis for many types of cancer, including acute promyelocytic leukemias [4], prostate cancer [5] and some types of melanomas [7]. An important feature of arsenic treatment is the quite narrow window of concentrations $(1-10 \mu \mathrm{M})$ when this agent may induce apoptosis by change in the pattern of specific gene expression. At higher doses of arsenite $(>10 \mu \mathrm{M})$, strong necrosis starts to substitute apoptosis of cancer cells, diminishing usefulness of this treatment. Two critical enzyme targets, which are suppressed by arsenite treatment, are IKK $\beta$ [41] and JAK2 [58]. Inhibition of these enzymes results in a suppression of the NF- $\mathrm{B}$ and STAT3-mediated signaling, which downregulates gene expression of cFLIP, XIAP, CIAP and BclxL [2,59], proteins involved in the regulation of anti-apoptotic protection in the cell (Fig. 9). On the other hand, stimulation of growth factor receptors by arsenite accelerates the MAPK/JNK signaling pathways $[9,60]$ that activates cJun, cFos and ATF2 transcription factors, which control transcription of both TRAIL and TRAIL-R1/R2 genes [26,27] (see Fig. 9). Finally, activation of Rac1 and Rac1-NADPH oxidase activity by sodium arsenite initiates a massive production of ROS in cells [61], which is followed by oxidative stress [62], mitochondrial dysfunction and an induction of mitochondrial death pathway in some melanoma lines. In addition, subsequent genotoxic stress also occurs, as well as an upregulation of p53 protein levels and p53-dependent gene expression, such as BAX and TRAIL-R2 [39,63-65], which is accompanied by an increase in apoptosis and secondary necrosis. Furthermore, levels of necrotic death are dramatically increased at arsenite doses higher than $10 \mu \mathrm{M}$. Arsenite also affects cytoskeleton functions and promotes TRAIL-R1/R2 translocation from the intracellular pools to cell surface, as it was observed in the present study. The existence of intracellular pools of TRAIL-R1/R2 and a suppression of receptor translocation in some cancer cell lines have been previously described in several publications [66,67].

Surprisingly, many cell types (both normal and cancer) are relatively resistant to cytotoxic effects of low doses of sodium arsenite, thereby making it possible to use this agent as a regulator of cell signaling pathways in different types of combined treatment. Indeed, our previous [7] and current studies indicate that most melanoma are relatively resistant to arsenite treatment (1-10 $\mu \mathrm{M})$ and, at best, may develop a modest TRAIL-mediated apoptosis. However, arsenite induced profound changes of susceptibility of melanoma to exogenous recombinant TRAIL by upregulation of TRAIL-R1 and R2 surface expression and JNK-cJun-mediated downregulation of cFLIP levels. Published reports indicate that myeloma cells display synergistic activation of TRAILmediated apoptosis by arsenic $[44,45]$. However, we observed largely additive effects of such combined treatment. Never-

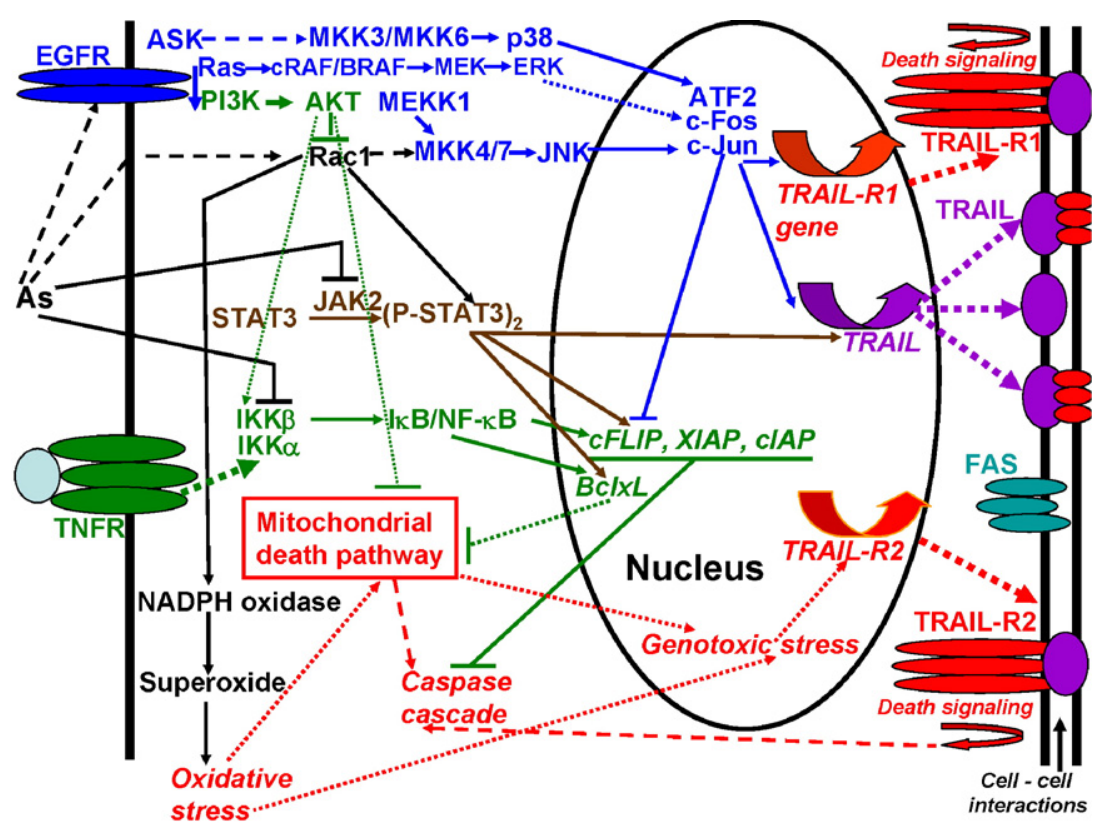

Fig. 9 - A schematic illustration of the principal signaling pathways that are affected by sodium arsenite (As) treatment. Arsenite accelerates activation of the MAPK pathways at the EGFR that finally upregulates MAPK p38, ERK and JNK and the correspondent transcription factors of the AP-1 family: ATF2, c-Fos, c-Jun. Arsenite suppresses the JAK2-STAT3 and IKK-NF- $\kappa B$ signaling pathways and downregulates expression of STAT3- and NF- $\kappa$ B-dependent anti-apoptotic genes. The third direction of the arsenic effects is linked with Rac1-NADPH oxidase activation, production ROS (superoxide), induction oxidative and genotoxic stress and initiation of the mitochondrial death pathway in some melanoma lines. At low doses (1-10 $\mu \mathrm{M})$, arsenite preferentially plays a role of the regulator of cell signaling and gene expression, inducing expression and translocation to the cell surface TRAIL and its receptor-1 and receptor-2. This creates conditions for an induction of the TRAIL-mediated death pathway via paracrine mechanism and dramatically increases a susceptibility of arsenite-treated cells to the exogenous TRAIL. See additional explanation and references in the text. 
theless, it overcame a partial resistance to TRAIL-mediated apoptosis, which has been demonstrated for some types of tumors, including melanomas. Many attempts have been made to increase a sensitivity of cancer cells to TRAIL using natural or pharmacological inhibitors of NF-кB, STAT3, AKT and COX-2 [46]. Mechanistically, given that arsenite inhibits NF- $\kappa$ B and STAT3 activation [7,41], this agent may be an effective inhibitor of cancer cell survival when used in combination with recombinant TRAIL, as was observed in the present study.

Numerous investigations performed on different types of cancer cells established anti-apoptotic role of both forms of cFLIP ( cFLIP $_{\mathrm{S}}$ and cFLIP $_{\mathrm{L}}$ ) in TRAIL- and FasL-mediated apoptosis $[13,14,18,19]$. However, CFLIP $_{\mathrm{L}}$ may perform antagonistic functions in the cell since its expression at lower levels actually supported caspase- 8 activation and cell death [20]. In the present study, we further confirmed antiapoptotic role of $\mathrm{CFLIP}_{\mathrm{L}}$ in melanomas, including melanoma cells with a deficiency of the mitochondrial death pathway (such as OM431 and SW1), and demonstrated that arsenite treatment dramatically downregulated cFLIP promoter activity and expression via negative regulation by the JNK-cJun pathway. A recent study has described a JNK-mediated protein degradation of $\mathrm{CFLIP}_{\mathrm{L}}$ through activation of the E3 protein ligase Itch [51], confirming the important role of $\mathrm{MAPK} / \mathrm{JNK}$ in the regulation of $\mathrm{CFLIP}_{\mathrm{L}}$ protein stability.

NF-кB-dependent control of cFLIP expression [42,49] appeared to be indirect (because the CFLIP promoter has no functional $\kappa \mathrm{B}$ sites) and may be based on NF- $\kappa \mathrm{B}$-dependent expression of COX-2 [50], which, in turn, may inhibit JNK and positively regulate CFLIP promoter activity. However, the COX2-prostaglandin E2 pathway possess multiple targets in the cell, in addition to regulation of cFLIP expression. COX-2 suppression dramatically increased sensitivity to sodium arsenite treatment in human melanomas due to both cFLIP downregulation and upregulation of surface expression FasL [48], increasing levels of FasL-mediated apoptosis.

Finally, the PI3K-AKT pathway that operates at many levels of cell survival is involved in the positive regulation of NF- $\kappa \mathrm{B}, \mathrm{COX}-2$ [54] and negative regulation of TRAIL [68] and TRAIL-R surface expression in melanomas, as we observed in the present study. On the other hand, treatment of melanoma cells with TRAIL in combination with the inhibitor of the PI3K-AKT pathway LY204002 substantially increased levels of cell death, especially necrotic death. In spite of the close similarity in the induction of death signaling via Fas and TRAIL-R pathways, there were remarkable differences in the regulation of expression and intracellular trafficking of endogenous FasL and TRAIL. One of these features was opposite effects of COX-2 suppression on arsenite-induced changes in TRAIL and FasL surface expression. A more complete understanding of the mechanism behind such differences will permit more accurate choice for induction of apoptosis in cancer cells.

In summary, finding regulators of cell signaling with opposite effects on the promoter activity of TRAIL and CFLIP is an intriguing prospect aim. In this respect, our investigation that JNK-cJun serves as a negative regulator of CFLIP and a positive regulator of TRAIL expression may open new possibilities in the anticancer therapy.

\section{Acknowledgments}

We thank Drs. Z. Ronai, V. Adler and S. Y. Fuchs and for discussion; Drs. M. Herlyn, O. Fodstad and L. B. Owen-Schaub for the melanoma cell lines; Drs. R. Davis, M. Karin, H. Wajant, W. S. El-Deiry, A. Chan, J. Hiscott and S.-Y. Sun for plasmid constructs; Dr. R. F. Qiao for monitoring tumor growth; and Dr. M. A. Partridge for critical reading of the manuscript. This work was supported by NIH Grant ES 11804, Superfund Grant P42 ES 10349, Environmental Center Grant P30 ES 09089 and NIEHS Center Pilot Award.

\section{R E F E R E N C E S}

[1] C. Perlis, M. Herlyn, Recent advances in melanoma biology, Oncologist 9 (2004) 182-187.

[2] K.M. Debatin, P.H. Krammer, Death receptors in chemotherapy and cancer, Oncogene 23 (2004) 2950-2966.

[3] J.C. Reed, M. Pellecchia, Apoptosis-based therapies for hematologic malignancies, Blood 106 (2005) 408-418.

[4] Z.X. Shen, G.Q. Chen, J.H. Ni, X.S. Li, S.M. Xiong, Q.Y. Qiu, J. Zhu, W. Tang, G.L. Sun, K.Q. Yang, Y. Chen, L. Zhou, Z.W. Fang, Y.T. Wang, J. Ma, P. Zhang, T.D. Zhang, S.J. Chen, Z. Chen, Z.Y. Wang, Use of arsenic trioxide (As2O3) in the treatment of acute promyelocytic leukemia (APL): II. Clinical efficacy and pharmacokinetics in relapsed patients, Blood 89 (1997) 3354-3360.

[5] M. Lu, L. Xia, D. Luo, S. Waxman, Y. Jing, Dual effects of glutathione-S-transferase pi on As2O3 action in prostate cancer cells: enhancement of growth inhibition and inhibition of apoptosis, Oncogene 23 (2004) 3945-3952.

[6] K.B. Kim, A.Y. Bedikian, L.H. Camacho, N.E. Papadopoulos, C. McCullough, A phase II trial of arsenic trioxide in patients with metastatic melanoma, Cancer 104 (2005) 1687-1692.

[7] V.N. Ivanov, T.K. Hei, Arsenite sensitizes human melanomas to apoptosis via tumor necrosis factor alpha-mediated pathway, J. Biol. Chem. 279 (2004) 22747-22758.

[8] V.N. Ivanov, T.K. Hei, Combined treatment with EGFR inhibitors and arsenite upregulated apoptosis in human EGFR-positive melanomas: a role of suppression of the PI3K-AKT pathway, Oncogene 24 (2005) 616-626.

[9] A.M. Bode, Z. Dong, The paradox of arsenic: molecular mechanisms of cell transformation and chemotherapeutic effects, Crit. Rev. Oncol./Hematol. 42 (2002) 5-24.

[10] A. Ashkenazi, Targeting death and decoy receptors of the tumour-necrosis factor superfamily, Nat. Rev., Cancer 2 (2002) 420-430.

[11] T.S. Griffith, W.A. Chin, G.C. Jackson, D.H. Lynch, M.Z. Kubin, Intracellular regulation of TRAIL-induced apoptosis in human melanoma cells, J. Immunol. 161 (1998) 2833-2840.

[12] P. Hersey, X.D. Zhang, How melanoma cells evade trail-induced apoptosis, Nat. Rev., Cancer 1 (2001) 142-150.

[13] J.H. Song, D.K. Song, M. Herlyn, K.C. Petruk, C. Hao, Cisplatin down-regulation of cellular Fas-associated death domain-like interleukin-1beta-converting enzyme-like inhibitory proteins to restore tumor necrosis factor-related apoptosis-inducing ligand-induced apoptosis in human melanoma cells, Clin. Cancer Res. 9 (2003) 4255-4266.

[14] C. Xiao, B.F. Yang, J.H. Song, H. Schulman, L. Li, C. Hao, Inhibition of CaMKII-mediated c-FLIP expression sensitizes malignant melanoma cells to TRAIL-induced apoptosis, Exp. Cell Res. 304 (2005) 244-255.

[15] H. Wajant, J. Gerspach, K. Pfizenmaier, Tumor therapeutics by 
design: targeting and activation of death receptors, Cytokine Growth Factor Rev. 16 (2005) 55-76.

[16] M. Karin, A. Lin, NF-kappaB at the crossroads of life and death, Nat. Immunol. 3 (2002) 221-227.

[17] A. Krueger, S. Baumann, P.H. Krammer, S. Kirchhoff, FLICE-inhibitory proteins: regulators of death receptor-mediated apoptosis, Mol. Cell. Biol. 21 (2001) 8247-8254.

[18] D.A. Sharp, D.A. Lawrence, A. Ashkenazi, Selective knockdown of the long variant of cellular FLICE inhibitory protein augments death receptor-mediated caspase-8 activation and apoptosis, J. Biol. Chem. 80 (2005) 19401-19409.

[19] M. Chawla-Sarkar, S.I. Bae, F.J. Reu, B.S. Jacobs, D.J. Lindner, E.C. Borden, Downregulation of Bcl-2, FLIP or IAPs (XIAP and survivin) by siRNAs sensitizes resistant melanoma cells to Apo2L/TRAIL-induced apoptosis, Cell Death Differ. 11 (2004) 915-923.

[20] D.W. Chang, Z. Xing, Y. Pan, A. Algeciras-Schimnich, B.C. Barnhart, S. Yaish-Ohad, M.E. Peter, X. Yang, C-FLIP(L) is a dual function regulator for caspase-8 activation and CD95-mediated apoptosis, EMBO J. 21 (2002) 3704-3714.

[21] R.C. Budd, W.C. Yeh, J. Tschopp, cFLIP regulation of lymphocyte activation and development, Nat. Rev., Immunol. 6 (2006) 196-204.

[22] V.N. Ivanov, A. Bhoumik, M. Krasilnikov, R. Raz, L.B. Owen-Schaub, D. Levy, C.M. Horvath, Z. Ronai, Cooperation between STAT3 and c-jun suppresses Fas transcription, Mol. Cell 7 (2001) 517-528.

[23] K. Satyamoorthy, E. DeJesus, A.J. Linnenbach, B. Kraj, D.L. Kornreich, S. Rendle, D.E. Elder, M. Herlyn, Melanoma cell lines from different stages of progression and their biological and molecular analyses, Melanoma Res. 7 (Suppl 2) (1997) S35-S42.

[24] H. van Dam, S. Huguier, K. Kooistra, J. Baguet, E. Vial, A.J. van der Eb, P. Herrlich, P. Angel, M. Castellazzi, Autocrine growth and anchorage independence: two complementing Jun-controlled genetic programs of cellular transformation, Genes Dev. 12 (1998) 1227-1239.

[25] Z. Wen, Z. Zhong, J.E. Darnell Jr., Maximal activation of transcription by Stat1 and Stat3 requires both tyrosine and serine phosphorylation, Cell 82 (1995) 241-250.

[26] T.M. Baetu, H. Kwon, S. Sharma, N. Grandvaux, J. Hiscott, Disruption of NF-kappaB signaling reveals a novel role for NF-kappaB in the regulation of TNF-related apoptosis-inducing ligand expression, J. Immunol. 167 (2001) 3164-3173.

[27] B. Guan, P. Yue, R. Lotan, S.Y. Sun, Evidence that the human death receptor 4 is regulated by activator protein 1 , Oncogene 21 (2002) 3121-3129.

[28] T. Bartke, D. Siegmund, N. Peters, M. Reichwein, F. Henkler, P. Scheurich, H. Wajant, p53 upregulates cFLIP, inhibits transcription of NF-kappaB-regulated genes and induces caspase-8-independent cell death in DLD-1 cells, Oncogene 20 (2001) 571-580.

[29] M.S. Ricci, Z. Jin, M. Dews, D. Yu, A. Thomas-Tikhonenko, D.T. Dicker, W.S. El-Deiry, Direct repression of FLIP expression by c-myc is a major determinant of TRAIL sensitivity, Mol. Cell. Biol. 24 (2004) 8541-8555.

[30] J.A. Brockman, D.C. Scherer, T.A. McKinsey, S.M. Hall, X. Qi, W.Y. Lee, D.W. Ballard, Coupling of a signal response domain in I kappa B alpha to multiple pathways for NF-kappa B activation, Mol. Cell. Biol. 15 (1995) 2809-2818.

[31] E. Zandi, D.M. Rothwarf, M. Delhase, M. Hayakawa, M. Karin, The IkappaB kinase complex (IKK) contains two kinase subunits, IKKalpha and IKKbeta, necessary for IkappaB phosphorylation and NF-kappaB activation, Cell 91 (1997) 243-252.

[32] A. Minden, A. Lin, M. McMahon, C. Lange-Carter, B. Derijard, R.J. Davis, G.L. Johnson, M. Karin, Differential activation of
ERK and JNK mitogen-activated protein kinases by Raf-1 and MEKK, Science 266 (1994) 1719-1723.

[33] C. Tournier, A.J. Whitmarsh, J. Cavanagh, T. Barrett, R.J. Davis, The MKK7 gene encodes a group of c-Jun NH2-terminal kinase kinases, Mol. Cell. Biol. 19 (1999) 1569-1581.

[34] L. Butterfield, B. Storey, L. Maas, L.E. Heasley, c-Jun NH2-terminal kinase regulation of the apoptotic response of small cell lung cancer cells to ultraviolet radiation, J. Biol. Chem. 272 (1997) 10110-10116.

[35] P.H. Brown, T.K. Chen, M.J. Birrer, Mechanism of action of a dominant-negative mutant of c-Jun, Oncogene 9 (1994) 791-799.

[36] R. Catlett-Falcone, T.H. Landowski, M.M. Oshiro, J. Turkson, A. Levitzki, R. Savino, G. Ciliberto, L. Moscinski, J.L. Fernandez-Luna, G. Nunez, W.S. Dalton, R. Jove, Constitutive activation of Stat 3 signaling confers resistance to apoptosis in human U266 myeloma cells, Immunity 10 (1999) 105-115.

[37] J.F. Bromberg, M.H. Wrzeszczynska, G. Devgan, Y. Zhao, R.G. Pestell, C. Albanese, J.E. Darnell Jr., Stat3 as an oncogene, Cell 98 (1999) 295-303.

[38] Z.X. Shen, Z.Z. Shi, J. Fang, B.W. Gu, J.M. Li, Y.M. Zhu, J.Y. Shi, P.Z. Zheng, H. Yan, Y.F. Liu, Y. Chen, Y. Shen, W. Wu, W. Tang, S. Waxman, H. De The, Z.Y. Wang, S.J. Chen, Z. Chen, All-trans retinoic acid/As2O3 combination yields a high quality remission and survival in newly diagnosed acute promyelocytic leukemia, Proc. Natl. Acad. Sci. U. S. A. 101 (2004) 5328-5335.

[39] R. Ravi, G.C. Bedi, L.W. Engstrom, Q. Zeng, B. Mookerjee, C. Gelinas, E.J. Fuchs, A. Bedi, Regulation of death receptor expression and TRAIL/Apo2L-induced apoptosis by NF-kappaB, Nat. Cell Biol. 3 (2001) 409-416.

[40] A.V. Franco, X.D. Zhang, E. Van Berkel, J.E. Sanders, X.Y. Zhang, W.D. Thomas, T. Nguyen, P. Hersey, The role of NF-kappa B in TNF-related apoptosis-inducing ligand (TRAIL)-induced apoptosis of melanoma cells, J. Immunol. 166 (2001) 5337-5345.

[41] P. Kapahi, T. Takahashi, G. Natoli, S.R. Adams, Y. Chen, R.Y. Tsien, M. Karin, Inhibition of NF-kappa B activation by arsenite through reaction with a critical cysteine in the activation loop of Ikappa B kinase, J. Biol. Chem. 275 (2000) 36062-36066.

[42] S. Kreuz, D. Siegmund, P. Scheurich, H. Wajant, NF-kappaB inducers upregulate cFLIP, a cycloheximide-sensitive inhibitor of death receptor signaling, Mol. Cell. Biol. 21 (2001) 3964-3973.

[43] Q. Wang, Y. Ji, X. Wang, B.M. Evers, Isolation and molecular characterization of the $5^{\prime}$-upstream region of the human TRAIL gene, Biochem. Biophys. Res. Commun. 76 (2000) 466-471.

[44] Q. Liu, S. Hilsenbeck, Y. Gazitt, Arsenic trioxide-induced apoptosis in myeloma cells: p53-dependent G1 or G2/M cell cycle arrest, activation of caspase-8 or caspase-9, and synergy with APO2/TRAIL, Blood 101 (2003) 4078-4087.

[45] E. Szegezdi, S. Cahill, M. Meyer, M. O’Dwyer, A. Samali, TRAIL sensitisation by arsenic trioxide is caspase- 8 dependent and involves modulation of death receptor components and Akt, Br. J. Cancer 94 (2006) 398-406.

[46] M. Karin, Y. Cao, F.R. Greten, Z.W. Li, NF-kappaB in cancer: from innocent bystander to major culprit, Nat. Rev., Cancer 2 (2002) 301-310.

[47] M. Krasilnikov, V.N. Ivanov, J. Dong, Z. Ronai, ERK and PI3K negatively regulate STAT-transcriptional activities in human melanoma cells: implications towards sensitization to apoptosis, Oncogene 22 (2003) 4092-4101.

[48] V.N. Ivanov, T.K. Hei, Dual treatment with COX-2 inhibitor and sodium arsenite leads to induction of surface Fas Ligand expression and Fas-Ligand-mediated apoptosis in human melanoma cells, Exp. Cell Res. 312 (2006) 1401-1417. 
[49] O. Micheau, S. Lens, O. Gaide, K. Alevizopoulos, J. Tschopp, NF-kappaB signals induce the expression of c-FLIP, Mol. Cell. Biol. 21 (2001) 5299-5305.

[50] T. Kanekura, S. Goorha, K. Kirtikara, L.R. Ballou, The involvement of NF-kappaB in the constitutive overexpression of cyclooxygenase-2 in cyclooxygenase-1 null cells, Biochim. Biophys. Acta 1542 (2002) 14-22.

[51] L. Chang, H. Kamata, G. Solinas, J.L. Luo, S. Maeda, K. Venuprasad, Y.C. Liu, M. Karin, The E3 ubiquitin ligase itch couples JNK activation to TNFalpha-induced cell death by inducing c-FLIP(L) turnover, Cell 124 (2006) 601-613.

[52] L. Xu, L. Zhang, Y. Yi, H.K. Kang, S.K. Datta, Human lupus T cells resist inactivation and escape death by upregulating COX-2, Nat. Med. 10 (2004) 411-415.

[53] B. Miller, Y.W. Chang, A. Sorokin, Cyclooxygenase 2 inhibits SAPK activation in neuronal apoptosis, Biochem. Biophys. Res. Commun. 300 (2003) 884-888.

[54] I. Vivanco, C.L. Sawyers, The phosphatidylinositol 3-Kinase AKT pathway in human cancer, Nat. Rev., Cancer 2 (2002) 489-501.

[55] V.N. Ivanov, M. Krasilnikov, Z. Ronai, Regulation of Fas expression by STAT3 and c-Jun is mediated by phosphatidylinositol 3-kinase-AKT signaling, J. Biol. Chem. 277 (2002) 4932-4944.

[56] A. Brunet, A. Bonni, M.J. Zigmond, M.Z. Lin, P. Juo, L.S. Hu, M.J. Anderson, K.C. Arden, J. Blenis, M.E. Greenberg, Akt promotes cell survival by phosphorylating and inhibiting a Forkhead transcription factor, Cell 96 (1999) 857-868.

[57] A. Bhoumik, T.G. Huang, V. Ivanov, L. Gangi, R.F. Qiao, S.L. Woo, S.H. Chen, Z. Ronai, An ATF2-derived peptide sensitizes melanomas to apoptosis and inhibits their growth and metastasis, J. Clin. Invest. 110 (2002) 643-650.

[58] H.Y. Cheng, P. Li, M. David, T.E. Smithgall, L. Feng, M.W. Lieberman, Arsenic inhibition of the JAK-STAT pathway, Oncogene 23 (2004) 3603-3612.
[59] H. Schulze-Bergkamen, P.H. Krammer, Apoptosis in cancer-Implications for therapy, Semin. Oncol. 31 (2004) 90-119.

[60] M. Cavigelli, W.W. Li, A. Lin, B. Su, K. Yoshioka, M. Karin, The tumor promoter arsenite stimulates AP-1 activity by inhibiting a JNK phosphatase, EMBO J. 15 (1996) 6269-6279.

[61] K.R. Smith, L.R. Klei, A. Barchowsky, Arsenite stimulates plasma membrane NADPH oxidase in vascular endothelial cells, Am. J. Physiol.: Lung Cell Mol. Physiol. 280 (2001) L442-L449.

[62] T.K. Hei, S.X. Liu, C. Waldren, Mutagenicity of arsenic in mammalian cells: role of reactive oxygen species, Proc. Natl. Acad. Sci. U. S. A. 95 (1998) 8103-8107.

[63] E.R. Sauter, R. Takemoto, S. Litwin, M. Herlyn, p53 alone or in combination with antisense cyclin D1 induces apoptosis and reduces tumor size in human melanoma, Cancer Gene. Ther. 9 (2002) 807-812.

[64] J.S. Fridman, S.W. Lowe, Control of apoptosis by p53, Oncogene 22 (2003) 9030-9040.

[65] Z. Jin, W.S. El-Deiry, Overview of cell death signaling pathways, Cancer Biol. Ther. 4 (2005) 139-163.

[66] X.D. Zhang, A.V. Franco, T. Nguyen, C.P. Gray, P. Hersey, Differential localization and regulation of death and decoy receptors for TNF-related apoptosis-inducing ligand (TRAIL) in human melanoma cells, J. Immunol. 164 (2000) 3961-3970.

[67] Z. Jin, E.R. McDonald III, D.T. Dicker, W.S. El-Deiry, Deficient tumor necrosis factor-related apoptosis-inducing ligand (TRAIL) death receptor transport to the cell surface in human colon cancer cells selected for resistance to TRAIL-induced apoptosis, J. Biol. Chem. 279 (2004) 35829-35839.

[68] Q. Wang, X. Wang, A. Hernandez, M.R. Hellmich, Z. Gatalica, B.M. Evers, Regulation of TRAIL expression by the phosphatidylinositol 3-kinase/Akt/GSK-3 pathway in human colon cancer cells, J. Biol. Chem. 277 (2002) 36602-36610. 